\title{
Exact integration scheme for planewave-enriched partition of unity finite element method to solve the Helmholtz problem
}

\author{
Subhajit Banerjee, N. Sukumar* \\ Department of Civil and Environmental Engineering, University of California, Davis, CA 95616, USA
}

\begin{abstract}
In this paper, we present an exact integration scheme to compute highly oscillatory integrals that appear in the solution of the two-dimensional Helmholtz problem using the planewave-enriched partition of unity finite element method. In the proposed scheme, such oscillatory integrals are computed by a recursive application of the divergence theorem, eventually expressing the integrals in terms of evaluations of the corresponding integrands at the nodes of the finite element mesh. The number of such function evaluations is independent of the wave number $k$, which permits the scheme to be used for arbitrary high values of $k$. We consider finite element meshes with unstructured triangular and structured rectangular elements, and present numerical results for three canonical benchmark Helmholtz problems to demonstrate the accuracy and efficacy of the method.

Keywords: Helmholtz equation, planewave enrichment, partition of unity finite element method, highly oscillatory integrals, divergence theorem, exact integration
\end{abstract}

\section{Introduction}

In the field of computational acoustics and electrodynamics, the problem of time harmonic wave scattered by bounded obstacles is of central importance. In mathematical physics this phenomenon is condensed in the framework of scattering theory. Scattering theory also finds its use in many applied disciplines such as: medical and seismic imaging, nondestructive testing, radar crosssection prediction, acoustic noise barrier, waveguides, etc. Use of high frequency time-harmonic waves offers distinct advantages in these applications, resulting in a proportionate time-harmonic response. The spatially varying component of this time-harmonic response is governed by the Helmholtz equation.

Consider a problem domain $\Omega \subset \mathbb{R}^{2}$ with $\boldsymbol{x} \equiv(x, y) \in \Omega$ and time $t \in[0, T] \subset \mathbb{R}^{+}$. Also let $\psi(\boldsymbol{x}, t)=u(\boldsymbol{x}) e^{-i \omega t}$ be a time-harmonic propagating disturbance with $i=\sqrt{-1}$, angular frequency $\omega$, and complex-valued spatially varying component $u(\boldsymbol{x})$. The scalar Helmholtz equation (in homogeneous form) governing $u(\boldsymbol{x})$ is:

$$
-\nabla^{2} u(x)-k^{2} u(x)=0,
$$

where $k=\omega / c$ is the constant wave number with $c$ denoting the wave velocity.

${ }^{*}$ Corresponding author

Email addresses: jitbanerjee@ucdavis .edu (Subhajit Banerjee), nsukumar@ucdavis.edu (N. Sukumar) 
Galerkin finite element method (FEM) is one of the most popular schemes to numerically solve the Helmholtz problem. For the Helmholtz equation in (1), it is well-known [1, 2] that the robustness of Galerkin FEM is compromised for higher values of $k$. This non-robust behavior is due to the so-called pollution effect. Moreover, for the Helmholtz equation (1), on a domain with characteristic length $L$ and wavelength $\lambda=2 \pi / k$, the dimensionless parameter $\lambda / L$ dictates the nature of the solution: $\lambda / L>>1$ induces oscillations. Accurate representation of such oscillatory solutions using piecewise-polynomial finite element approximation spaces demands refined meshes. Typically, in one-dimension, Galerkin FEM adopts the convention of $h \approx \lambda / 10$ [3], where $h$ is the representative element size. This requires a large number of degrees of freedom in the high wave number regime $(k>>1)$, and hence to reduce computational costs high-order numerical schemes are used.

Several high-order schemes have been proposed over the past two decades to solve (11). Most of these methods are based on the use of non-polynomial oscillatory basis functions that augment the piecewise polynomial finite element (FE) approximation space. Among these, we can mention:

1. Galerkin least-squares finite element method (GLS) [4],

2. partition of unity finite element method (PUFEM) [5-7] or the generalized finite element method (GFEM) [8-10],

3. ultra-weak variational formulation (UWVF) [11, 12],

4. discontinuous enrichment method (DEM) [13, 14],

5. particle partition of unity method (P-PUM) [15, 16],

6. plane wave discontinuous Galerkin (PWDG) method [17], and

7. planewave virtual element method (PW-VEM) [18]

as some of the most promising methods. The PUFEM/GFEM offers the advantages of interelement continuity of the interpolated variables, and is a superset of the underlying FE approximation space. In these methods, the FE approximation space is enriched with special, problemdependent, non-polynomial or Fourier basis set. For the Helmholtz equation in two dimensions, the most frequently used enrichment functions are the system of planewaves. In polar coordinates, these functions are represented as:

$$
\exp \left(i k r \cos \left(\theta-\theta_{n}\right)\right) ; \quad n=0,1, \ldots,
$$

where the angles $\theta_{n} \in[0,2 \pi]$ represent the different directions of propagation of these planewaves. The planewaves are pasted locally on the FE mesh using set of nodally defined and compactly supported partition-of-unity (PU) functions. For a wave number $k$, planewave enrichments are more conveniently represented in Cartesian coordinates as [5, 8, 19]

$$
\left\{\psi_{k, q}^{n}=\exp \left[i k\left(x \cos \frac{2 \pi n}{q}+y \sin \frac{2 \pi n}{q}\right)\right]\right\}_{n=0}^{q-1},
$$

where $q$ is the number of enrichment functions at a given node of the FE mesh. Due to the presence of oscillatory basis functions, the stiffness matrix that stems from the PUFEM contains several oscillatory integrals of the form:

$$
\mathcal{I}_{[m, n]}=\int_{\Omega} x^{m} y^{n} \exp \left(i \boldsymbol{k}_{n} \cdot \boldsymbol{x}\right) d \boldsymbol{x}, \quad \boldsymbol{k}_{n}=k\left(\cos \theta_{n} \boldsymbol{e}_{1}+\sin \theta_{n} \boldsymbol{e}_{2}\right)
$$


for a planewave enrichment function pointing at an angle $\theta_{n}$ with respect to some reference direction and with $\left\{\boldsymbol{e}_{i}\right\}_{i=1}^{2}$ denoting unit vectors along the coordinate directions. Accurate and efficient evaluation of such integrals is crucial to the success of the planewave-enriched PUFEM.

Interpolatory quadrature schemes assume an underlying polynomial representation of the oscillatory integrand in (4). Such a representation is only accurate for moderate values of $k$. However, for $k>>1$ (see Fig. 1), the integrand in (4) becomes highly oscillatory. Adopting existing interpolatory quadrature schemes leads to use of large number of quadrature points. In $d$-dimensions, due to the Cartesian product construction of interpolatory cubature, $O\left(N^{d}\right)$ function evaluations are required. This is popularly known as the curse of dimensionality. In Reference [20], the authors report the use of up to $120 \times 120$ Gauss quadrature points per element in two dimensions. Often, this entails greater computational cost than that of solving the system of linear equations, and hence use of interpolatory cubature rules for high wave number Helmholtz problem compromises the efficiency of the PUFEM.

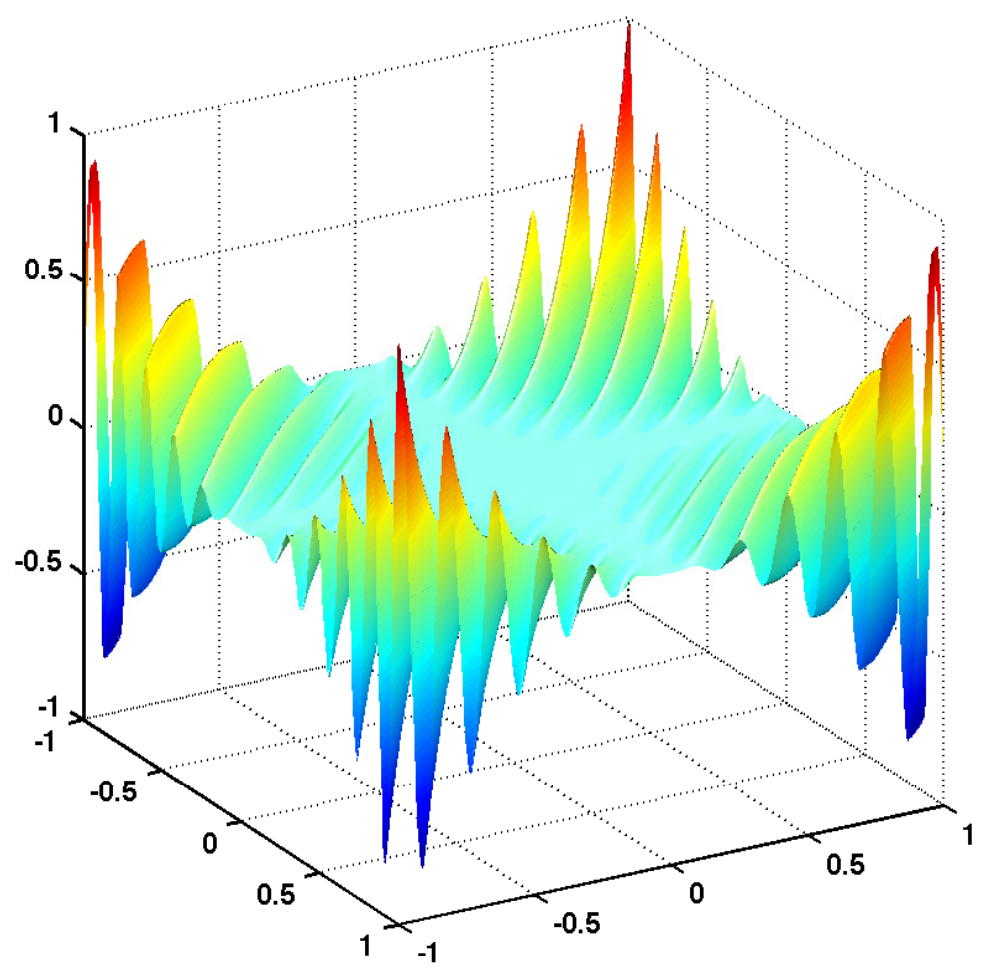

Figure 1: Oscillatory integrand: real part of $x^{2} y^{2} \exp (i \boldsymbol{k} \cdot \boldsymbol{x})$ on the biunit square with $\boldsymbol{k}=50\left[\cos (\pi / 4) \boldsymbol{e}_{1}+\sin (\pi / 4) \boldsymbol{e}_{2}\right]$.

Numerical computation of highly oscillatory integrals is an active area of research. The problem of computing an integral of the form

$$
\mathcal{I}[f, \omega]=\int_{\Omega} f(\boldsymbol{x}) \exp (i \omega g(\boldsymbol{x})) d \boldsymbol{x},
$$

where $\Omega \subset \mathbb{R}^{d}, f$ and the oscillator $g$ are non-oscillatory, smooth functions, has far-reaching applications beyond the PUFEM. The generic techniques to compute (5) fall within three broad categories, namely asymptotic methods, Filon-type methods [21, 22], and Levin-type collocation 
methods [23]. For a comprehensive overview of such integrals, the reader is referred to References [24-26] and the references therein. In the context of planewave-enriched PUFEM, the issue of accurate numerical integration has been discussed in References [6, 27]. Ortiz and Sanchez [28] presented a method where the oscillatory integrands are rotated using a unitary transformation to obtain an equivalent one-dimensional oscillatory integrand. Bettes et al. [29] devised a semianalytical integration scheme, which is based on considering finite number of terms in the infinite series expansion of the exponential terms. The scheme is compared to Gauss quadrature points in the range of 1-120. In this paper, we draw on the scheme developed in References [30, 31] for integrating homogeneous functions over a polytope using the generalized Stokes's theorem. We devise a scheme to extend this to the more involved case of oscillatory integrals of the form (4).

The remainder of this paper is organized as follows. In Section 2, we present the exact integration scheme and demonstrate its efficacy. Then in Section 3, we describe the essentials of the PUFEM to solve the Helmholtz problem. In Section 4, we derive the oscillatory integrals that appear in the system matrices of the PUFEM, and the implementational aspects of the method are discussed in Section 4.1. For rectangular finite elements, we show the advantages of using the parent coordinate system to incorporate the new scheme. In Section 5, we demonstrate the capabilities of the proposed scheme by solving three canonical Helmholtz problems borrowed from scattering theory, and close with some final remarks in Section 6 .

\section{Exact scheme to compute oscillatory integrals}

In this section we present an exact analytical scheme to compute integrals of the type shown in (4). The relevant derivations are given for the two-dimensional case.

\subsection{Arbitrary polygon}

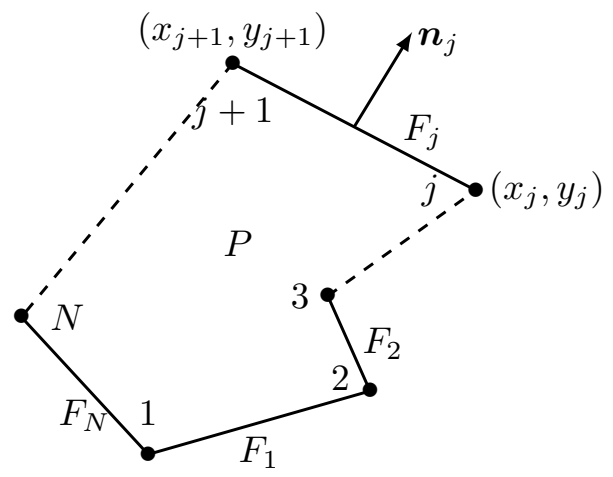

Figure 2: Simple polygon $P$.

Referring to Fig. 2, consider the closed polygon $P \subset \mathbb{R}^{2}$ that is defined by its $N$ vertices $\left(x_{1}, y_{1}\right), \ldots,\left(x_{N}, y_{N}\right)$. We use $\partial P$ to denote the boundary of $P$, which is defined by $N$ boundary edges $F_{1}, \ldots, F_{N}$. The boundary edge $F_{j}$ is formed by connecting the vertices $\left(x_{j}, y_{j}\right)$ and $\left(x_{j+1}, y_{j+1}\right)$ and we set $\left(x_{N+1}, y_{N+1}\right) \equiv\left(x_{1}, y_{1}\right)$ to form the closed polygon. The boundary edge $F_{j}$ is represented by the linear relation

$$
\boldsymbol{a}_{j} \cdot \boldsymbol{x}=b_{j}
$$


for some vector $\boldsymbol{a}_{j}$ and scalar $b_{j}$ that are dependent on $\left(x_{j}, y_{j}\right)$ and $\left(x_{j+1}, y_{j+1}\right)$. Now, consider (4) once again and let

$$
g(\boldsymbol{x}) \equiv g(x, y)=x^{m} y^{n} \exp \left(i \boldsymbol{k}_{n} \cdot \boldsymbol{x}\right)
$$

with its gradient given by

$$
\nabla g=i \boldsymbol{k}_{n} g+m x^{m-1} y^{n} \exp \left(i \boldsymbol{k}_{n} \cdot \boldsymbol{x}\right) \boldsymbol{e}_{1}+n x^{m} y^{n-1} \exp \left(i \boldsymbol{k}_{n} \cdot \boldsymbol{x}\right) \boldsymbol{e}_{2} .
$$

For the polygonal domain $P$, (4) becomes

$$
\mathcal{I}_{[m, n]}^{P}=\int_{P} g d x
$$

We now introduce the divergence theorem in the following form:

$$
\int_{P}(\nabla \cdot \boldsymbol{X}) g d \boldsymbol{x}+\int_{P} \boldsymbol{X} \cdot \nabla g d \boldsymbol{x}=\int_{\partial P}(\boldsymbol{X} \cdot \boldsymbol{n}) g d \Gamma=\sum_{j=1}^{N} \int_{F_{j}}\left(\boldsymbol{X} \cdot \boldsymbol{n}_{j}\right) g d \Gamma
$$

where $\boldsymbol{X}$ is an arbitrary vector field defined in $P, d \Gamma$ is the differential line element on $\partial P$, and $\boldsymbol{n}$ is the unit outward normal vector on $\partial P$. Furthermore, we note that $\boldsymbol{n}_{j}=\boldsymbol{a}_{j} /\left\|\boldsymbol{a}_{j}\right\|$ (see Fig. 2), the normal to the boundary edge $F_{j}$. Now, referring to (10), we let $\boldsymbol{X}=\boldsymbol{\zeta}=\zeta_{1} \boldsymbol{e}_{1}+\zeta_{2} \boldsymbol{e}_{2}$ be a constant vector field. With this and using (8), (10) simplifies to

$i \zeta \cdot \boldsymbol{k}_{n} \int_{P} g d \boldsymbol{x}+m \zeta_{1} \int_{P} x^{m-1} y^{n} \exp \left(i \boldsymbol{k}_{n} \cdot \boldsymbol{x}\right) d \boldsymbol{x}+n \zeta_{2} \int_{P} x^{m} y^{n-1} \exp \left(i \boldsymbol{k}_{n} \cdot \boldsymbol{x}\right) d \boldsymbol{x}=\sum_{j=1}^{N}\left(\zeta \cdot \frac{\boldsymbol{a}_{j}}{\left\|\boldsymbol{a}_{j}\right\|}\right) \int_{F_{j}} g d \Gamma$.

Rearranging the last equation and using the definition of $g$ from (7) yields

$$
\begin{aligned}
i \zeta \cdot \boldsymbol{k}_{n} \int_{P} x^{m} y^{n} \exp \left(i \boldsymbol{k}_{n} \cdot \boldsymbol{x}\right) d \boldsymbol{x}= & \sum_{j=1}^{N}\left(\zeta \cdot \frac{\boldsymbol{a}_{j}}{\left\|\boldsymbol{a}_{j}\right\|}\right) \int_{F_{j}} x^{m} y^{n} \exp \left(i \boldsymbol{k}_{n} \cdot \boldsymbol{x}\right) d \Gamma \\
& -m \zeta_{1} \int_{P} x^{m-1} y^{n} \exp \left(i \boldsymbol{k}_{n} \cdot \boldsymbol{x}\right) d \boldsymbol{x} \\
& -n \zeta_{2} \int_{P} x^{m} y^{n-1} \exp \left(i \boldsymbol{k}_{n} \cdot \boldsymbol{x}\right) d \boldsymbol{x} .
\end{aligned}
$$

At this point, we introduce the notation

$$
\mathcal{I}_{[m, n]}^{F_{j}}=\int_{F_{j}} g d \Gamma=\int_{F_{j}} x^{m} y^{n} \exp \left(i \boldsymbol{k}_{n} \cdot \boldsymbol{x}\right) d \Gamma
$$

so that $(11)$ is rewritten as

$$
\mathcal{I}_{[m, n]}^{P}=\frac{1}{i \boldsymbol{\zeta} \cdot \boldsymbol{k}_{n}}\left[\sum_{j=1}^{N}\left(\boldsymbol{\zeta} \cdot \frac{\boldsymbol{a}_{j}}{\left\|\boldsymbol{a}_{j}\right\|}\right) \mathcal{I}_{[m, n]}^{F_{j}}-m \zeta_{1} \mathcal{I}_{[(m-1), n]}^{P}-n \zeta_{2} \mathcal{I}_{[m,(n-1)]}^{P}\right]
$$


In (13), observe that to compute $\mathcal{I}_{[m, n]}^{P}$ one must compute two similar oscillatory domain integrals $\mathcal{I}_{[(m-1), n]}^{P}$ and $\mathcal{I}_{[m,(n-1)]}^{P}$ over the polygon $P$ along with $N$ oscillatory line integrals $\mathcal{I}_{[m, n]}^{F_{j}}$ $(j=1, \ldots, N)$ on the respective bounding edges $F_{j}$. This recursive structure is exploited in the present work and is presented in Algorithm 1. The termination condition of the recursion to compute the domain integral is achieved when

$$
\mathcal{I}_{[0,0]}^{P}=\int_{P} \exp \left(i \boldsymbol{k}_{n} \cdot \boldsymbol{x}\right) d \boldsymbol{x}
$$

is reached. Next, we describe the computation of $\mathcal{I}_{[0,0]}^{P}$ as well as all the line integrals of the form $\mathcal{I}_{[p, q]}^{F_{j}}$ generated at every level of recursion, namely $p=m,(m-1), \ldots, 0$ and $q=n,(n-1), \ldots, 0$.

\subsubsection{Computing $\mathcal{I}_{[0,0]}^{P}$}

To compute $\mathcal{I}_{[0,0]}^{P}$ we proceed along similar lines as above and also make use of the identity

$$
\nabla\left[\exp \left(i \boldsymbol{k}_{n} \cdot \boldsymbol{x}\right)\right]=i \boldsymbol{k}_{n} \exp \left(i \boldsymbol{k}_{n} \cdot \boldsymbol{x}\right)
$$

to obtain

$$
\mathcal{I}_{[0,0]}^{P}=\int_{P} \exp \left(i \boldsymbol{k}_{n} \cdot \boldsymbol{x}\right) d \boldsymbol{x}=\frac{1}{i \zeta \cdot \boldsymbol{k}_{n}} \sum_{j=1}^{N}\left(\zeta \cdot \frac{\boldsymbol{a}_{j}}{\left\|\boldsymbol{a}_{j}\right\|}\right) \int_{F_{j}} \exp \left(i \boldsymbol{k}_{n} \cdot \boldsymbol{x}\right) d \Gamma .
$$

Following (12), we can write

$$
\mathcal{I}_{[0,0]}^{F_{j}}=\int_{F_{j}} \exp \left(i \boldsymbol{k}_{n} \cdot \boldsymbol{x}\right) d \Gamma
$$

and (14) becomes

$$
\mathcal{I}_{[0,0]}^{P}=\frac{1}{i \boldsymbol{\zeta} \cdot \boldsymbol{k}_{n}} \sum_{j=1}^{N}\left(\zeta \cdot \frac{\boldsymbol{a}_{j}}{\left\|\boldsymbol{a}_{j}\right\|}\right) \mathcal{I}_{[0,0]}^{F_{j}} .
$$

$\mathcal{I}_{[0,0]}^{P}$ in $[15)$ is computed using Algorithm 3 .

Lastly, we point out that since $\zeta$ is an arbitrary constant vector field it is possible that $\boldsymbol{\zeta} \cdot \boldsymbol{k}_{n}=0$ can arise. This leads to the undesirable division-by-zero problem in (13) and (15). To avoid this pitfall, we always choose $\zeta=\boldsymbol{k}_{n} /\left\|\boldsymbol{k}_{n}\right\|$.

\subsubsection{Computing $\mathcal{I}_{[m, n]}^{F_{j}}$}

We also need to compute the oscillatory line integrals $\mathcal{I}_{[m, n]}^{F_{j}}$ of the form shown in (12) as well as the line integrals $\mathcal{I}_{[0,0]}^{F_{j}}$ appearing in $(15)$ for $j=1, \ldots, N$. To this end, we invoke a variant of the divergence theorem in (10) to convert the line integrals to integrals on a set of measure zero.

Referring to Fig. 3, let $F_{j i}=F_{j} \cap F_{i}$ be the vertex formed at the point of intersection of edges $F_{j}$ and $F_{i}$. Also, let $\boldsymbol{n}_{j i}$ be the unit vector in two-dimensions that lies on $F_{j}$ and is normal to $F_{j i}$. This is trivially achieved as any vector passing through the vertex $F_{j i}$ is normal to it. A constant unit vector field $\boldsymbol{t}_{j}=\left(t_{j}\right)_{1} \boldsymbol{e}_{1}+\left(t_{j}\right)_{2} \boldsymbol{e}_{2}$ that lies on the edge $F_{j}$ is also introduced. The vector field $\boldsymbol{t}_{j}$ plays the same role as $\zeta$ that appears in $(11)$. 


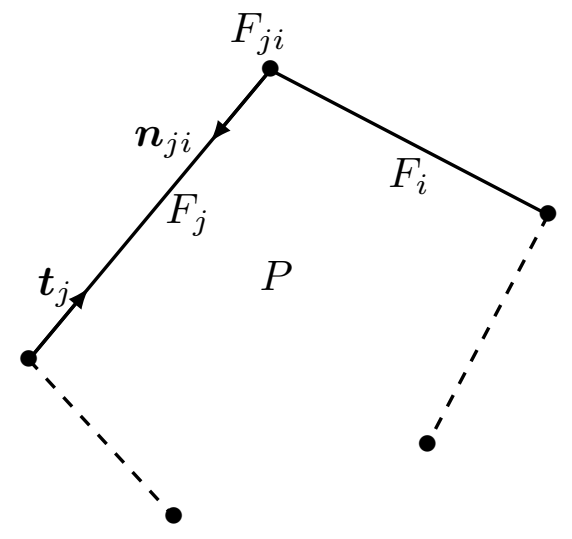

Figure 3: Boundaries of polygon $P$.

Following steps similar to those in Section 2.1, the line integrals $\mathcal{I}_{[m, n]}^{F_{j}}$ are then recursively computed as

$$
\mathcal{I}_{[m, n]}^{F_{j}}=\frac{1}{i \boldsymbol{t}_{j} \cdot \boldsymbol{k}_{n}}\left[\sum_{l \neq j}\left(\boldsymbol{t}_{j} \cdot \boldsymbol{n}_{j l}\right) \int_{F_{j l}} x^{m} y^{n} \exp \left(i \boldsymbol{k}_{n} \cdot \boldsymbol{x}\right) d \mu-\left(t_{j}\right)_{1} m \mathcal{I}_{[(m-1), n]}^{F_{j}}-\left(t_{j}\right)_{2} n \mathcal{I}_{[m,(n-1)]}^{F_{j}}\right]
$$

In (16), $\int_{F_{j l}} x^{m} y^{n} \exp \left(i \boldsymbol{k}_{n} \cdot \boldsymbol{x}\right) d \mu$ is the value of the integrand at the vertex $F_{j l}$, and $\boldsymbol{t}_{j} \cdot \boldsymbol{n}_{j l}$ that can only assume \pm 1 (see Fig. 3) provides its sign. Lastly, letting $\boldsymbol{x}_{j+1}=x_{j+1} \boldsymbol{e}_{1}+y_{j+1} \boldsymbol{e}_{2}$ and $\boldsymbol{x}_{j}=x_{j} \boldsymbol{e}_{1}+y_{j} \boldsymbol{e}_{2}$, the termination condition $\mathcal{I}_{[0,0]}^{F_{j}}$ (appears in (15) as well) is expressed as

$$
\begin{aligned}
\mathcal{I}_{[0,0]}^{F_{j}} & =\frac{1}{i \boldsymbol{t}_{j} \cdot \boldsymbol{k}_{n}}\left[\sum_{l \neq j}\left(\boldsymbol{t}_{j} \cdot \boldsymbol{n}_{j l}\right) \int_{F_{j l}} \exp \left(i \boldsymbol{k}_{n} \cdot \boldsymbol{x}\right) d \mu\right] \\
& =\frac{1}{i \boldsymbol{t}_{j} \cdot \boldsymbol{k}_{n}}\left[\exp \left(i \boldsymbol{k}_{n} \cdot \boldsymbol{x}_{j+1}\right)-\exp \left(i \boldsymbol{k}_{n} \cdot \boldsymbol{x}_{j}\right)\right] .
\end{aligned}
$$

The pseudo-code to implement the recursive scheme in (16) and (17) is presented in Algorithm 2 .

\subsubsection{Treatment of special case: $\boldsymbol{t}_{j} \cdot \boldsymbol{k}_{n}=0$}

Unlike the case of (13) wherein we avoided the division-by-zero issue by carefully selecting $\boldsymbol{\zeta}=\boldsymbol{k}_{n} /\left\|\boldsymbol{k}_{n}\right\|$, we cannot make this choice. In (16), since we have $\boldsymbol{t}_{j}$ bound on $F_{j}$ and $\boldsymbol{k}_{n}$ is dependent on the enrichment direction, there can be degenerate cases for different choices of $\boldsymbol{t}_{j}$ for a given $\boldsymbol{k}_{n}$ such that $\boldsymbol{t}_{j} \cdot \boldsymbol{k}_{n}=0$. In these cases, we resort to analytical means for evaluating $\mathcal{I}_{[m, n]}^{F_{j}}$. To this end, the integral in (12) is transformed using a parameter $s \in[0,1]$. Recall from Fig. 2 that the edge $F_{j}$ is formed by connecting the nodes located at $\left(x_{j}, y_{j}\right)$ and $\left(x_{j+1}, y_{j+1}\right)$. Hence, we rewrite (12) in terms of the parameter $s$ as

$$
\mathcal{I}_{[m, n]}^{F_{j}}=\int_{0}^{1}\left[(1-s) x_{j}+s x_{j+1}\right]^{m}\left[(1-s) y_{j}+s y_{j+1}\right]^{n} \exp \left(i \boldsymbol{k}_{n} \cdot\left[(1-s) \boldsymbol{x}_{j}+s \boldsymbol{x}_{j+1}\right]\right) L_{j} d s,
$$


where $L_{k}$ is the length of the boundary edge $F_{k}$. Additionally, setting $\Delta x_{k}=\left(x_{k+1}-x_{k}\right), \Delta y_{k}=$ $\left(y_{k+1}-y_{k}\right)$, and $\Delta \boldsymbol{x}_{k}=\Delta x_{k} \boldsymbol{e}_{1}+\Delta y_{k} \boldsymbol{e}_{2},(18)$ simplifies to

$$
\begin{aligned}
\mathcal{I}_{[m, n]}^{F_{j}} & =\int_{0}^{1}\left(x_{j}+s \Delta x_{j}\right)^{m}\left(y_{j}+s \Delta y_{j}\right)^{n} \exp \left(i \boldsymbol{k}_{n} \cdot \Delta \boldsymbol{x}_{j} s\right) \exp \left(i \boldsymbol{k}_{n} \cdot \boldsymbol{x}_{j}\right) L_{j} d s \\
& =L_{j} \exp \left(i \boldsymbol{k}_{n} \cdot \boldsymbol{x}_{j}\right) \int_{0}^{1}\left(x_{j}+s \Delta x_{j}\right)^{m}\left(y_{j}+s \Delta y_{j}\right)^{n} \exp (i \mathcal{K} s) d s,
\end{aligned}
$$

where $\mathcal{K}=\boldsymbol{k}_{n} \cdot \Delta \boldsymbol{x}_{j}$. We use (19) on the boundary edges $F_{j}$ for which $\boldsymbol{t}_{j} \cdot \boldsymbol{k}_{n}=0$. But, in those cases $\mathcal{K}=\boldsymbol{k}_{n} \cdot \Delta \boldsymbol{x}_{j}=0$ as $\Delta \boldsymbol{x}_{j}$ is always aligned along $\boldsymbol{t}_{j}$. This results in nullifying the oscillatory part $\exp (i \mathcal{K} s)$ of (19). Hence, the following simpler expression is obtained:

$$
\begin{aligned}
\mathcal{I}_{[m, n]}^{F_{j}} & =L_{j} \exp \left(i \boldsymbol{k}_{n} \cdot \boldsymbol{x}_{j}\right) \int_{0}^{1}\left(x_{j}+s \Delta x_{j}\right)^{m}\left(y_{j}+s \Delta y_{j}\right)^{n} d s \\
& =L_{j} \exp \left(i \boldsymbol{k}_{n} \cdot \boldsymbol{x}_{j}\right) \sum_{\alpha=0}^{m} \sum_{\beta=0}^{n}\left[\left(\begin{array}{l}
m \\
\alpha
\end{array}\right)\left(\begin{array}{l}
n \\
\beta
\end{array}\right) \frac{x_{j}^{\alpha} y_{j}^{\beta} \Delta x_{j}^{m-\alpha} \Delta y_{j}^{n-\beta}}{m+n-(\alpha+\beta)+1}\right] .
\end{aligned}
$$

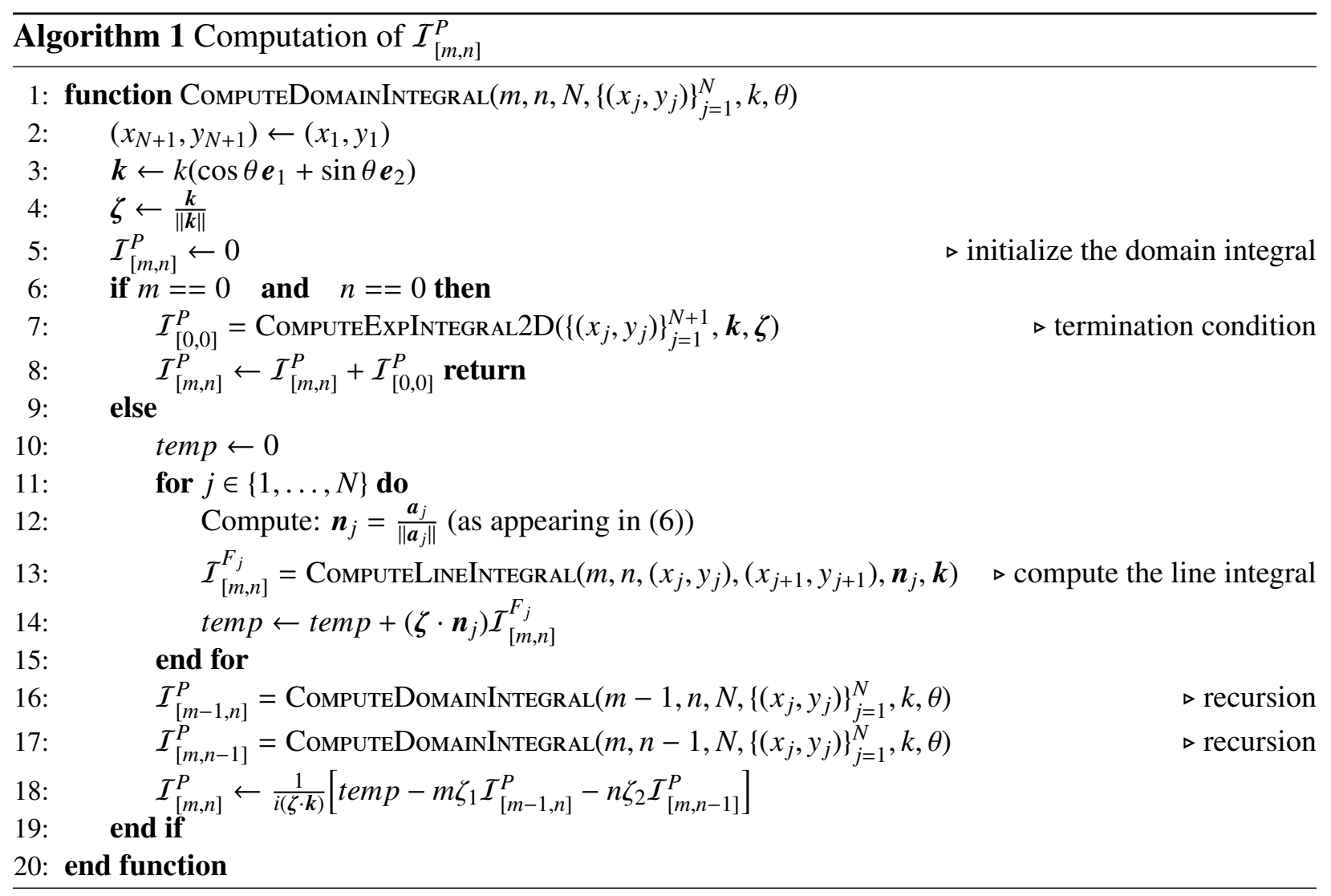



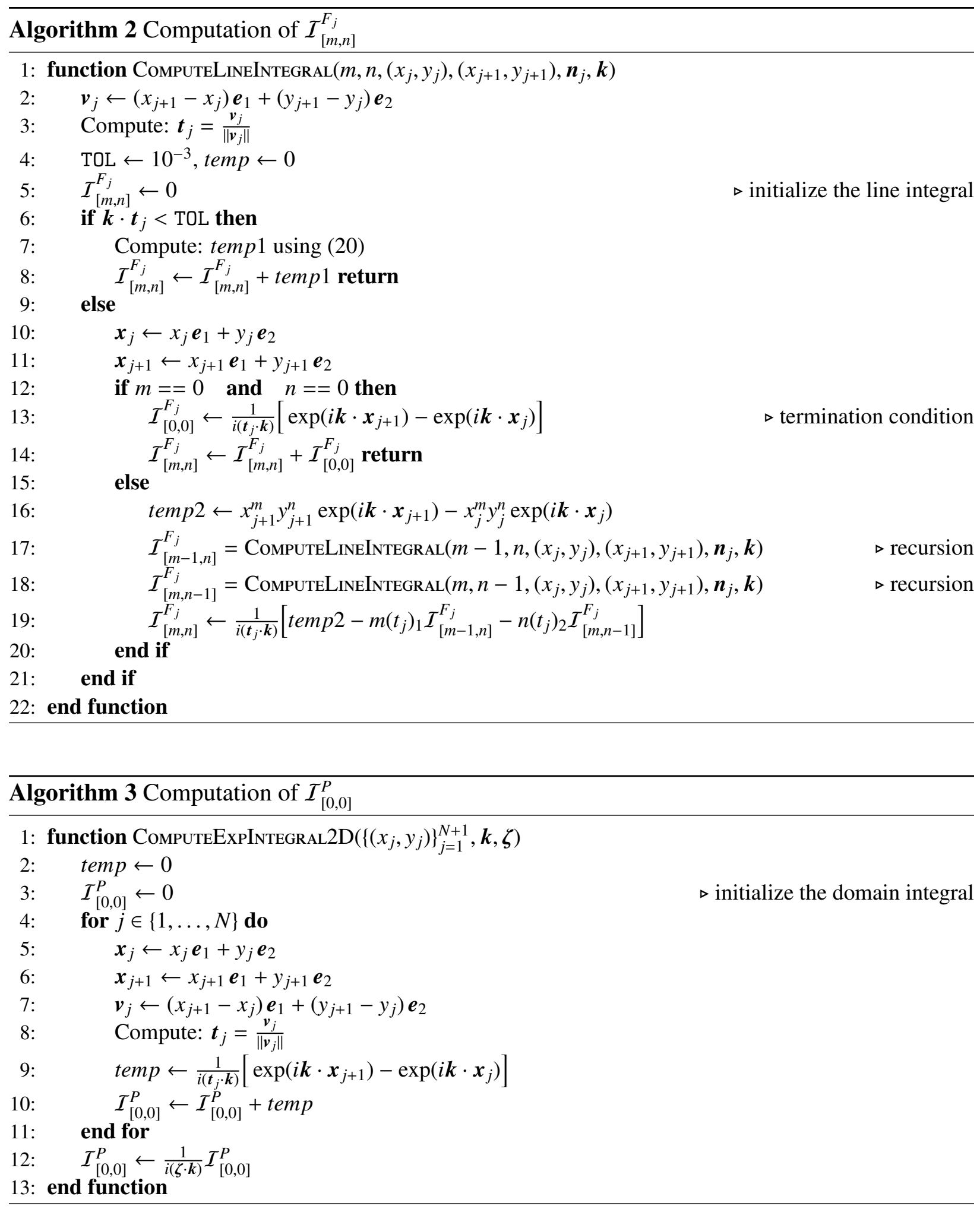

\section{Helmholtz problem}

In this section, we formulate the boundary-value problem corresponding to (1), and then using its weak form, the discrete equations for the PUFE are setup. 


\subsection{Strong and weak forms}

We consider the exterior problem corresponding to (1), which is formulated assuming $\Omega$ to be an unbounded domain. A typical Helmholtz problem in two dimensions often constitutes: a unit strength incident planewave $u_{I}(\boldsymbol{x})=\exp \left(i k \boldsymbol{p}_{I} \cdot \boldsymbol{x}\right)$, traveling along the direction given by the unit vector $\boldsymbol{p}_{I}$, and scattered off the boundary $\partial \Omega_{s}$ of a bounded scatter (obstacle) $\Omega_{s}$. We denote the scattered wave by $u_{S}(\boldsymbol{x})$. The problem definition is schematically shown in Fig. 4.

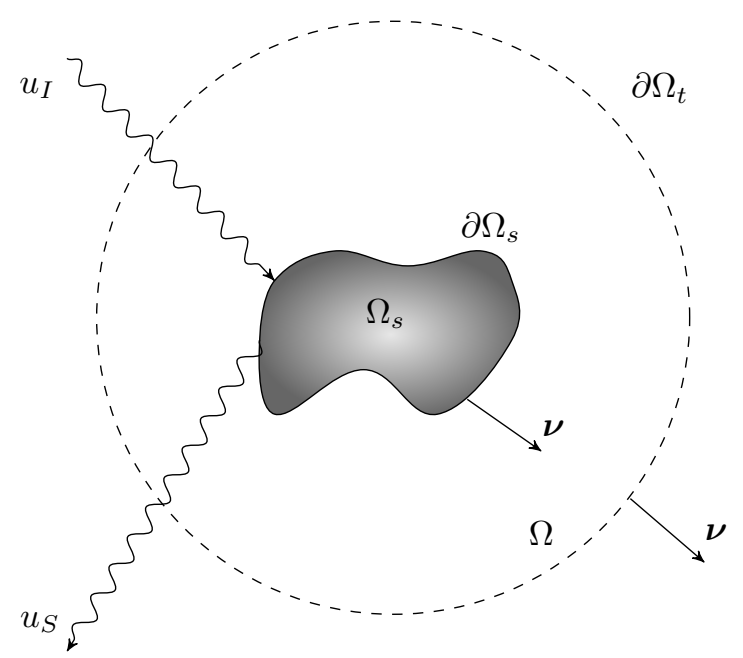

Figure 4: Problem domain $\Omega$ of a typical scattering problem in two-dimensions. $\partial \Omega_{s}$ represents the boundary of the scatter $\Omega_{s}$ and $\partial \Omega_{t}$ is the truncation boundary. $u_{I}$ and $u_{S}$ represent the incident and scattered waves, respectively. $v$ denotes the unit outward normal vector to the boundary $\partial \Omega=\partial \Omega_{s} \cup \partial \Omega_{t}$.

In this case, the total field $u(\boldsymbol{x})=u_{I}(\boldsymbol{x})+u_{S}(\boldsymbol{x})$ satisfies (1). Being a planewave, $u_{I}(\boldsymbol{x})$ also satisfies (1) and so does $u_{S}(\boldsymbol{x})$. The exterior problem is well-posed if the scattered wave is outgoing [3], i.e., the scattered wave is not reflected back from infinity. This amounts to $u_{S}$ satisfying the far-field (Sommerfeld radiation) condition:

$$
\lim _{r \rightarrow \infty} \sqrt{r}\left(\frac{\partial u_{S}}{\partial r}-i k u_{S}\right)=0
$$

where $r=\sqrt{\boldsymbol{x} \cdot \boldsymbol{x}}$. We truncate the infinite domain of the exterior problem to a finite one for making it amenable to finite-dimensional Galerkin-type approximations. To this end, we envelope the scatter $\Omega_{s}$ by the truncation boundary $\partial \Omega_{t}$ and denote $\partial \Omega=\partial \Omega_{s} \cup \partial \Omega_{t}$. To replicate the outgoing radiation condition on $\partial \Omega_{t}$ generally an absorbing boundary condition (ABC) is imposed [3].

For scattered wave $u_{S}$, the Helmholtz problem is stated as

$$
\begin{aligned}
-\nabla^{2} u_{S}-k^{2} u_{S} & =0 & & \text { in } \Omega \\
(1+Q) \frac{\partial u_{S}}{\partial v}+(1-Q) i k u_{S} & =g & & \text { on } \partial \Omega
\end{aligned}
$$

where $\boldsymbol{v}$ (see Fig. (4) is the outward normal vector to $\partial \Omega$ and $g$ is the source term defined on $\partial \Omega$. In [22), the constant $Q \in \mathbb{C}$ with $|Q|<1$, can be tuned to generate different types of boundary 
conditions. The corresponding weak form statement of (22) is: find $u \in H^{1}(\Omega)$ such that

$$
\begin{aligned}
a(w, u) & =\ell(w) \quad \forall w \in H^{1}(\Omega), \quad \text { where } \\
a(w, u) & =\int_{\Omega} \nabla w^{*} \cdot \nabla u d \Omega-k^{2} \int_{\Omega} w^{*} u d \Omega+i k\left(\frac{1-Q}{1+Q}\right) \int_{\partial \Omega} w^{*} u d \Gamma \\
\ell(w) & =\frac{1}{1+Q} \int_{\partial \Omega} w^{*} g d \Gamma,
\end{aligned}
$$

where $u$ and $w$ are in general complex-valued trial and test approximations.

\subsection{PUFE formulation}

Use of the PUFE scheme to solve high wave number Helmholtz problem was first proposed in Reference [5] and later extended in References [9, 10]. Typically, one starts by defining, at each node of the FE mesh, $q$ number of globally-supported planewave enrichment functions $\left\{\psi_{k, q}^{n}\right\}_{n=0}^{q-1}$ (see Fig. 6b) of the form shown in (3). These are usually uniformly spaced along the directions

$$
\left\{\left[\cos \left(\frac{2 \pi n}{q}\right) \boldsymbol{e}_{1}+\sin \left(\frac{2 \pi n}{q}\right) \boldsymbol{e}_{2}\right]\right\}_{n=0}^{q-1},
$$

which represent unit vectors pointing towards the direction of propagation of these planewaves. This is schematically shown in Fig. 5. In general, these enrichment functions need not be uniformly spaced and not all nodes of the FE mesh are required to be enriched with the same number of enrichment functions.

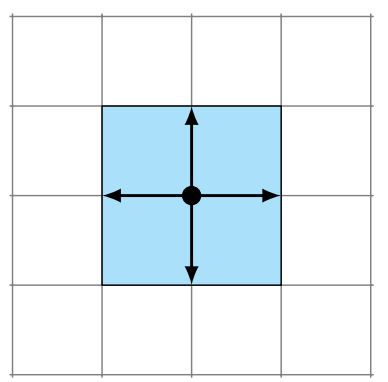

(a)

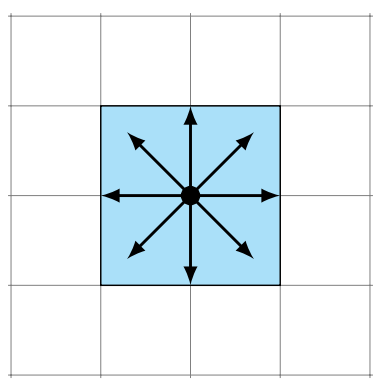

(b)

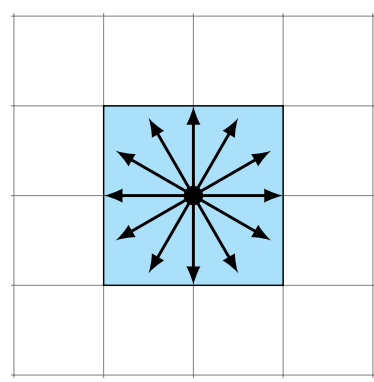

(c)

Figure 5: Uniformly-spaced enrichment directions at a finite element node (represented as filled, black circle) for (a) $q=4$, (b) $q=8$, and (c) $q=12$. The shaded regions represent the support of the nodal FE basis function.

The enrichment functions are pasted locally at each node using the finite element basis functions as the partition-of-unity functions subordinate to the FE mesh (see Fig. 6a). An enrichment function is shown in Fig. 6b and the compactly-supported partitioned enrichment function is constructed as the product of the FE basis function and the enrichment function (see Fig. 6c).

The PUFE approximation in element $e$ for wave number $k$ is:

$$
u_{e}^{h}(\boldsymbol{x})=\sum_{a=1}^{n e l} N_{a}(\boldsymbol{x}) \hat{u}_{a}+\sum_{b=1}^{n e l} N_{b}(\boldsymbol{x}) \sum_{n=0}^{q-1} \psi_{k, q}^{n}(\boldsymbol{x}) \hat{a}_{b n} .
$$




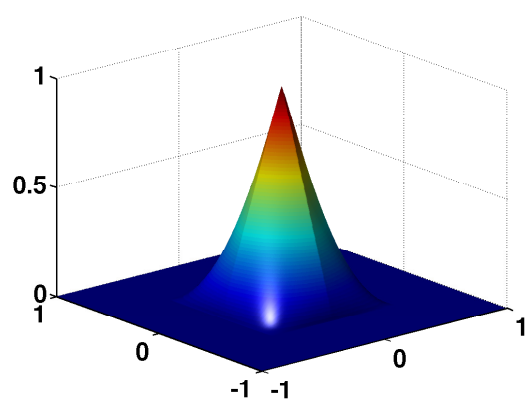

(a)

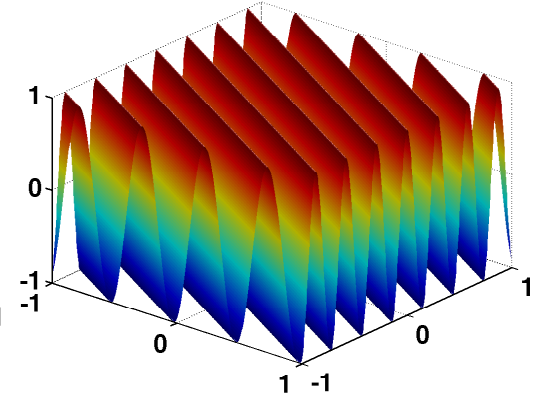

(b)

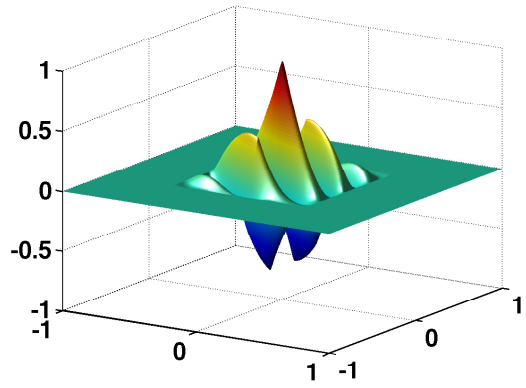

(c)

Figure 6: Plots of basis functions. (a) Bilinear finite element basis function for a node $b$ in the FE mesh; (b) Globallysupported planewave enrichment function $\psi_{k, q}^{n}$ for $k=20, n=6$, and $q=9$; and (c) Compactly-supported nonpolynomial partitioned enrichment function.

In (24), $N_{a}(\boldsymbol{x})$ is the finite element shape function at node $a, \hat{u}_{a}$ and $\hat{a}_{b n}$ are the degrees of freedom corresponding to the finite element and enriched basis functions, respectively, and nel is the number of nodes of element $e$. Finite-dimensional trial and test approximations of the form (24) are substituted in the weak form (23), and using the arbitrariness of the test coefficients, the discrete equations at the element-level are obtained. Finite element assembly procedures are used to obtain the global stiffness matrix and load vector.

\section{Oscillatory integrals in the stiffness matrix}

In this section, we derive the terms that appear in the PUFE stiffness matrix. The FE mesh consists of four-node bilinear quadrilateral or three-node linear triangular elements. We carry out the derivations in the parent coordinate system $(\xi, \eta)$. For a rectangular finite element $\Omega_{e}$, a point $(x, y) \in \Omega_{e}$ is transformed to $\Omega_{\square}=[-1,1]^{2}$ (biunit square) or $\Omega_{\Delta}=\{\xi, \eta \geq 0, \xi+\eta \leq 1\}$ (reference triangle). The PUFE approximation $u_{e}^{h}$ in (24) is rewritten as

$$
u_{e}^{h}(\boldsymbol{x}(\xi, \eta))=\boldsymbol{N}(\xi, \eta)^{\top} \hat{\boldsymbol{u}}_{e}+\boldsymbol{\Phi}(\xi, \eta)^{\top} \hat{\boldsymbol{a}}_{e}
$$

where $\boldsymbol{N}=\left[N_{1} \cdots N_{n e l}\right]^{\top}$ and $\boldsymbol{\Phi}=\left[N_{1} \psi_{k, q}^{0} \cdots N_{1} \psi_{k, q}^{q-1} N_{2} \psi_{k, q}^{0} \cdots N_{n e l} \psi_{k, q}^{q-1}\right]^{\top}$. We can write the element stiffness matrix $\boldsymbol{k}_{e}$ as

$$
\begin{aligned}
\boldsymbol{k}_{e} & =\int_{\hat{\Omega}}\left[\begin{array}{cc}
\frac{\partial \boldsymbol{N}}{\partial \xi} & \frac{\partial \boldsymbol{N}}{\partial \eta} \\
\frac{\partial \boldsymbol{\Phi}^{*}}{\partial \xi} & \frac{\partial \boldsymbol{\Phi}^{*}}{\partial \eta}
\end{array}\right] \boldsymbol{J}^{-\top} \boldsymbol{J}^{-1}\left[\begin{array}{cc}
\frac{\partial N^{\top}}{\partial \xi} & \frac{\partial \boldsymbol{\Phi}^{\top}}{\partial \xi} \\
\frac{\partial N^{\top}}{\partial \eta} & \frac{\partial \boldsymbol{\Phi}^{\top}}{\partial \eta}
\end{array}\right]|\boldsymbol{J}| d \hat{\boldsymbol{\Omega}}-k^{2} \int_{\hat{\Omega}}\left[\begin{array}{cc}
\boldsymbol{N} \boldsymbol{N}^{\top} & \boldsymbol{N}^{\top} \\
\boldsymbol{\Phi}^{*} \boldsymbol{N}^{\top} & \boldsymbol{\Phi}^{*} \boldsymbol{\Phi}^{\top}
\end{array}\right]|\boldsymbol{J}| d \hat{\boldsymbol{\Omega}} \\
& +i k\left(\frac{1-Q}{1+Q}\right) \int_{\hat{\Gamma}}\left[\begin{array}{ll}
\boldsymbol{N}_{1 d} \boldsymbol{N}_{1 d}^{\top} & \boldsymbol{N}_{1 d} \boldsymbol{\Phi}_{1 d}^{\top} \\
\boldsymbol{\Phi}_{1 d}^{*} \boldsymbol{N}_{1 d}^{\top} & \boldsymbol{\Phi}_{1 d}^{*} \boldsymbol{\Phi}_{1 d}^{\top}
\end{array}\right]|\boldsymbol{J}|_{1 d} d \hat{\Gamma} .
\end{aligned}
$$

In 26

$$
\hat{\Omega}=\left\{\begin{array}{ll}
\Omega_{\square} & \text { for quadrilateral FE } \\
\Omega_{\triangle} & \text { for triangular FE }
\end{array},\right.
$$

where $|\boldsymbol{J}|$ is the determinant of the Jacobian of the $\Omega_{e} \mapsto \hat{\Omega}$ transformation $\left(\boldsymbol{J}_{1 d}\right.$ is the corresponding Jacobian in one dimension), $\boldsymbol{N}_{1 d}$ and $\boldsymbol{\Phi}_{1 d}$ are the one-dimensional counterparts of $\boldsymbol{N}$ and $\boldsymbol{\Phi}$, 
respectively, and $\hat{\Gamma}$ is the one-dimensional linear parent element. The element load vector $\boldsymbol{f}_{e}$ is given by

$$
\boldsymbol{f}_{e}=\frac{1}{1+Q} \int_{\hat{\Gamma}} g\left[\begin{array}{l}
N_{1 d} \\
\boldsymbol{\Phi}_{1 d}^{*}
\end{array}\right]|\boldsymbol{J}|_{1 d} d \hat{\Gamma}
$$

The element stiffness matrix $\boldsymbol{k}_{e}$ is further partitioned into four block matrices, such that

$$
\boldsymbol{k}_{e}=\left[\begin{array}{c|c}
\left(\boldsymbol{k}_{e}\right)_{\mathrm{FE}-\mathrm{FE}} & \left(\boldsymbol{k}_{e}\right)_{\mathrm{PU}-\mathrm{FE}} \\
\hline\left(\boldsymbol{k}_{e}\right)_{\mathrm{FE}-\mathrm{PU}} & \left(\boldsymbol{k}_{e}\right)_{\mathrm{PU}-\mathrm{PU}}
\end{array}\right]
$$

Note that the integrals appearing in the block matrices $\left(\boldsymbol{k}_{e}\right)_{\mathrm{PU}-\mathrm{FE}},\left(\boldsymbol{k}_{e}\right)_{\mathrm{FE}-\mathrm{PU}}=\left(\boldsymbol{k}_{e}^{*}\right)_{\mathrm{PU}-\mathrm{FE}}^{\top}$, and $\left(\boldsymbol{k}_{e}\right)_{\mathrm{PU}-\mathrm{PU}}$ are oscillatory in nature with the form (in the parent coordinate system)

$$
\mathcal{I}_{[m, n]}=\int_{\hat{\Omega}} \xi^{m} \eta^{n} \exp (i \boldsymbol{k} \cdot \boldsymbol{\xi}) d \boldsymbol{\xi}
$$

whereas the non-oscillatory integrals in $\left(\boldsymbol{k}_{e}\right)_{\mathrm{FE}-\mathrm{FE}}$ are of the form

$$
I_{[p, q]}=\int_{\hat{\Omega}} \xi^{p} \eta^{q} d \boldsymbol{\xi}
$$

The element stiffness matrices $\boldsymbol{k}_{e}$ and the element force vectors $\boldsymbol{f}_{e}$ are assembled to construct the global stiffness matrix $\boldsymbol{K}$ and the global load vector $\boldsymbol{f}$. This results in the global system of linear equations, which is given by

$$
\begin{aligned}
& \boldsymbol{K} \boldsymbol{d}=\boldsymbol{f} \\
& \boldsymbol{K}=\left[\begin{array}{c|c}
(\boldsymbol{K})_{\mathrm{FE}-\mathrm{FE}} & (\boldsymbol{K})_{\mathrm{PU}-\mathrm{FE}} \\
\hline(\boldsymbol{K})_{\mathrm{FE}-\mathrm{PU}} & (\boldsymbol{K})_{\mathrm{PU}-\mathrm{PU}}
\end{array}\right], \\
& \boldsymbol{d}=\left[\hat{u}_{1} \cdots \hat{u}_{N} \hat{a}_{11} \hat{a}_{12} \cdots \hat{a}_{1 q} \hat{a}_{21} \cdots \hat{a}_{2 q} \cdots \cdots \hat{a}_{N 1} \cdots \hat{a}_{N q}\right]^{\top},
\end{aligned}
$$

where the stiffness matrix $\boldsymbol{K}$ has a $2 \times 2$ block-structure and $\boldsymbol{d}$, which contains the FE and enriched degrees of freedom, is consistent with the block-structure in (31b).

\subsection{Representation of planewave enrichments in parent coordinate system}

To obtain a closed-form expression for the stiffness integrals we represent $\psi_{k, q}^{n}$ in the parent coordinate system. Additional advantages of such a representation become apparent as we carry out the necessary derivations. To this end, let

$$
\boldsymbol{W}=\left[\psi_{k, q}^{0} \cdots \psi_{k, q}^{q-1}\right]^{\top} \equiv \exp \left[i k\left\{x \cos \left(\frac{2 \pi}{q} \boldsymbol{n}\right)+y \sin \left(\frac{2 \pi}{q} \boldsymbol{n}\right)\right\}\right]
$$

where $\boldsymbol{n}=[0 \cdots(q-1)]^{\top}$. 


\subsubsection{Biunit square}

We first consider the case of the biunit square $\Omega_{\square}$ (Fig. 7). Referring to Fig. 7, let $\Delta x=$ $\left(x_{2}^{e}-x_{1}^{e}\right)=\left(x_{3}^{e}-x_{4}^{e}\right)$ and $\Delta y=\left(y_{3}^{e}-y_{2}^{e}\right)=\left(y_{4}^{e}-y_{1}^{e}\right)$. We only consider regular Cartesian meshes, and therefore $\Delta x$ and $\Delta y$ are each the same for any finite element. The isoparametric formulation for the four-node element is:

$$
x(\xi, \eta)=\frac{1}{4} \sum_{a=1}^{4} x_{a}^{e}+\frac{\Delta x}{2} \xi, \quad y(\xi, \eta)=\frac{1}{4} \sum_{a=1}^{4} y_{a}^{e}+\frac{\Delta y}{2} \eta .
$$

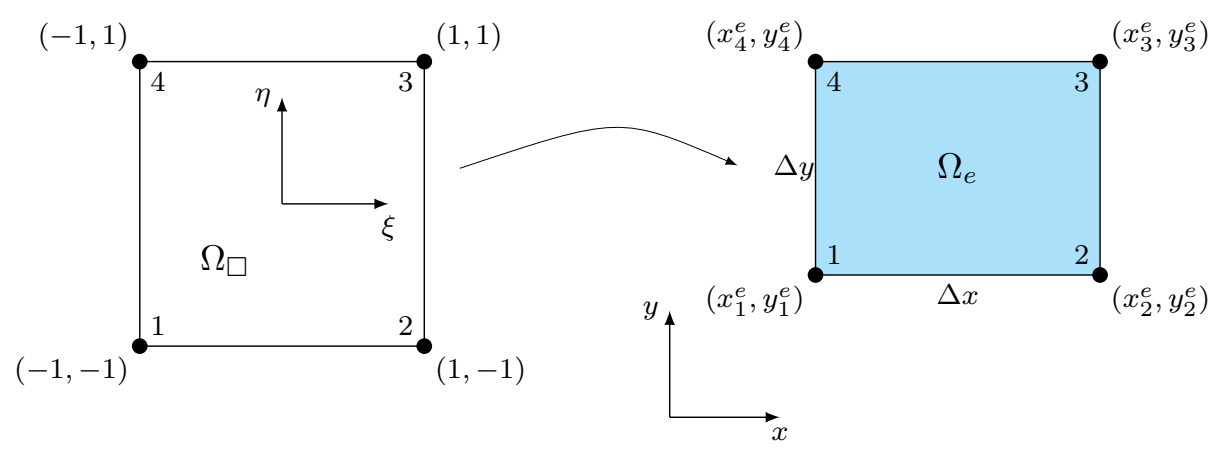

Figure 7: Affine mapping from four-node physical element $\Omega_{\square}$ to $\Omega_{e}$.

Using this transformation, $\boldsymbol{W}$ is now rewritten as

$$
\boldsymbol{W}=\exp \left[\frac{i k}{4}\left\{\sum_{a=1}^{4} x_{a}^{e} \cos \left(\frac{2 \pi}{q} \boldsymbol{n}\right)+\sum_{a=1}^{4} y_{a}^{e} \sin \left(\frac{2 \pi}{q} \boldsymbol{n}\right)\right\}\right] \exp \left[\frac{i k}{2}\left\{\Delta x \xi \cos \left(\frac{2 \pi}{q} \boldsymbol{n}\right)+\Delta y \eta \sin \left(\frac{2 \pi}{q} \boldsymbol{n}\right)\right\}\right] .
$$

Let the element dependent constant exponent in (32) be denoted by

$$
\gamma_{n}^{e}=\left[\frac{1}{4}\left\{\sum_{a=1}^{4} x_{a} \cos \left(\frac{2 \pi}{q} n\right)+\sum_{a=1}^{4} y_{a} \sin \left(\frac{2 \pi}{q} n\right)\right\}\right]
$$

Also, let $k_{\xi ; n}=\frac{k \Delta x}{2} \cos \left(\frac{2 \pi}{q} n\right), k_{\eta ; n}=\frac{k \Delta y}{2} \sin \left(\frac{2 \pi}{q} n\right)$, and $\boldsymbol{k}_{n}=\left[k_{\xi ; n} k_{\eta ; n}\right]^{\top}$ to rewrite (32) for enrichment direction $n$ as

$$
W_{n}^{e}=\exp \left(i k \gamma_{n}^{e}\right) \exp \left(i \boldsymbol{k}_{n} \cdot \boldsymbol{\xi}\right)
$$

for $n=0, \ldots,(q-1)$. Referring to 26$)$, the derivatives of $W_{n}^{e}$ can be expressed as

$$
\frac{\partial W_{n}^{e}}{\partial \xi}=i k_{\xi}^{n} W_{n}^{e}, \quad \frac{\partial W_{n}^{e}}{\partial \eta}=i k_{\eta}^{n} W_{n}^{e}
$$

for $n=0, \ldots,(q-1)$. Closed-form expressions for these integrals on $\Omega_{\square}$ are given in Appendix A.

For Cartesian meshes, the components of the local wave numbers $\boldsymbol{k}_{n}$, i.e., $k_{\xi ; n}$ and $k_{\eta ; n}$ are independent of the nodal coordinates of the element, and only depend on $k, \Delta x$, and $\Delta y$. The element-dependence is encapsulated in $\gamma_{n}^{e}$. This enables us to compute the required oscillatory integrals in (26) just once for each $n=0, \cdots,(q-1)$ using the nodal coordinates information of $\Omega_{\square}$, and the components of the local wave numbers $k_{\xi ; n}$ and $k_{\eta ; n}$. 


\subsubsection{Unit reference triangle}

We next consider the case of the unit reference triangle $\Omega_{\Delta}$ (see Fig. 8). The isoparametric formulation for the three-node triangular element is:

$$
x(\xi, \eta)=x_{1}^{e}+\left(x_{2}^{e}-x_{1}^{e}\right) \xi+\left(x_{3}^{e}-x_{1}^{e}\right) \eta, \quad y(\xi, \eta)=y_{1}^{e}+\left(y_{2}^{e}-y_{1}^{e}\right) \xi+\left(y_{3}^{e}-y_{1}^{e}\right) \eta .
$$

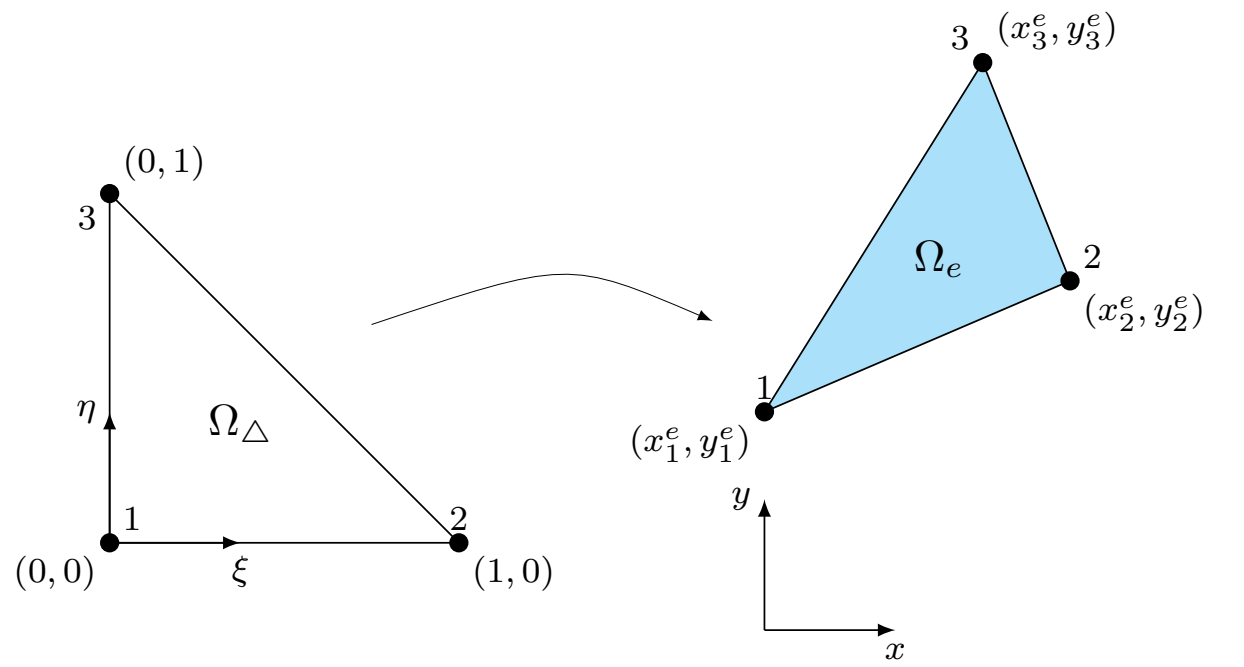

Figure 8: Affine mapping from three node physical element $\Omega_{\Delta}$ to $\Omega_{e}$.

As in the case of $\Omega_{\square}$, we proceed to represent the planewave enrichments in $\Omega_{\triangle}$. Using the transformation in (33), we obtain

$$
\begin{aligned}
& \boldsymbol{W}=\exp \left[i k\left\{x_{1}^{e} \cos \left(\frac{2 \pi}{q} \boldsymbol{n}\right)+y_{1}^{e} \sin \left(\frac{2 \pi}{q} \boldsymbol{n}\right)\right\}\right] \\
& \exp \left[i k\left\{\left\{\left(x_{2}^{e}-x_{1}^{e}\right) \cos \left(\frac{2 \pi}{q} \boldsymbol{n}\right)+\left(y_{2}^{e}-y_{1}^{e}\right) \sin \left(\frac{2 \pi}{q} \boldsymbol{n}\right)\right\} \xi+\left\{\left(x_{3}^{e}-x_{1}^{e}\right) \cos \left(\frac{2 \pi}{q} \boldsymbol{n}\right)+\left(y_{3}^{e}-y_{1}^{e}\right) \sin \left(\frac{2 \pi}{q} \boldsymbol{n}\right)\right\} \eta\right\}\right] .
\end{aligned}
$$

The element dependent constants

$$
\begin{aligned}
& \lambda_{\xi ; n}^{e}=\left(x_{2}^{e}-x_{1}^{e}\right) \cos \left(\frac{2 \pi}{q} n\right)+\left(y_{2}^{e}-y_{1}^{e}\right) \sin \left(\frac{2 \pi}{q} n\right), \\
& \lambda_{\eta ; n}^{e}=\left(x_{3}^{e}-x_{1}^{e}\right) \cos \left(\frac{2 \pi}{q} n\right)+\left(y_{3}^{e}-y_{1}^{e}\right) \sin \left(\frac{2 \pi}{q} n\right), \\
& \gamma_{n}^{e}=x_{1}^{e} \cos \left(\frac{2 \pi}{q} n\right)+y_{1}^{e} \sin \left(\frac{2 \pi}{q} n\right)
\end{aligned}
$$

are used to define the local wave numbers $k_{\xi ; n}^{e}=k \lambda_{\xi ; n}^{e}$ and $k_{\eta ; n}^{e}=k \lambda_{\eta ; n}^{e}$. Additionally, we let $\boldsymbol{k}_{n}^{e}=\left[\begin{array}{ll}k_{\xi ; n}^{e} & k_{\eta ; n}^{e}\end{array}\right]^{\top}$ to rewrite (34) for enrichment direction $n$ as

$$
W_{n}^{e}=\exp \left(i k \gamma_{n}^{e}\right) \exp \left(i \boldsymbol{k}_{n}^{e} \cdot \boldsymbol{\xi}\right)
$$

for $n=0, \ldots,(q-1)$. The partial derivatives of $W_{n}^{e}$ are

$$
\frac{\partial W_{n}^{e}}{\partial \xi}=i k \lambda_{\xi ; n}^{e} W_{n}^{e}=i k_{\xi ; n}^{e} W_{n}^{e}, \quad \frac{\partial W_{n}^{e}}{\partial \eta}=i k \lambda_{\eta ; n}^{e} W_{n}^{e}=i k_{\eta ; n}^{e} W_{n}^{e}
$$


for $n=0, \ldots,(q-1)$. Closed-form expressions for these integrals on $\Omega_{\triangle}$ are presented in Appendix B.

Unlike the case of $\Omega_{\square}$, for $\Omega_{\Delta}$ the local wave numbers $k_{\xi ; n}^{e}$ and $k_{\eta ; n}^{e}$ are now dependent on the nodal coordinates of $\Omega_{e}$. Thus, in spite of the geometry information for the recursive algorithm (Section 2) being fixed, the $\xi, \eta$ components of the local wave number change for each element. This renders computations on triangular meshes to be more expensive than those on Cartesian meshes.

\subsubsection{One-dimensional linear element}

The line integrals in (26) and (27) are

$$
\int_{\hat{\Gamma}}\left[\begin{array}{ll}
\boldsymbol{N}_{1 d} \boldsymbol{N}_{1 d}^{\top} & \boldsymbol{N}_{1 d} \boldsymbol{\Phi}_{1 d}^{\top} \\
\boldsymbol{\Phi}_{1 d}^{*} \boldsymbol{N}_{1 d}^{\top} & \boldsymbol{\Phi}_{1 d}^{*} \boldsymbol{\Phi}_{1 d}^{\top}
\end{array}\right]|\boldsymbol{J}|_{1 d} d \hat{\Gamma}, \quad \int_{\hat{\Gamma}} g\left\{\begin{array}{l}
\boldsymbol{N}_{1 d} \\
\boldsymbol{\Phi}_{1 d}^{*}
\end{array}\right\}|\boldsymbol{J}|_{1 d} d \hat{\Gamma},
$$

which are oscillatory in nature. To express these line integrals in parent coordinates, we consider a one-dimensional linear Lagrange element $\Gamma_{e}$ with nodal coordinates $\left\{x_{a}^{e_{1 d}}, y_{a}^{e_{1 d}}\right\}_{a=1}^{2}$. This gets mapped to the one-dimensional two-node biunit parent element $\hat{\Gamma}$ (see Fig. 9). The isoparametric map yields

$$
x(\xi)=\frac{1}{2} \sum_{a=1}^{2} x_{a}^{e_{1 d}}+\frac{\Delta x^{e_{1 d}}}{2} \xi, \quad y(\xi)=\frac{1}{2} \sum_{a=1}^{2} y_{a}^{e_{1 d}}+\frac{\Delta y^{e_{1 d}}}{2} \xi .
$$

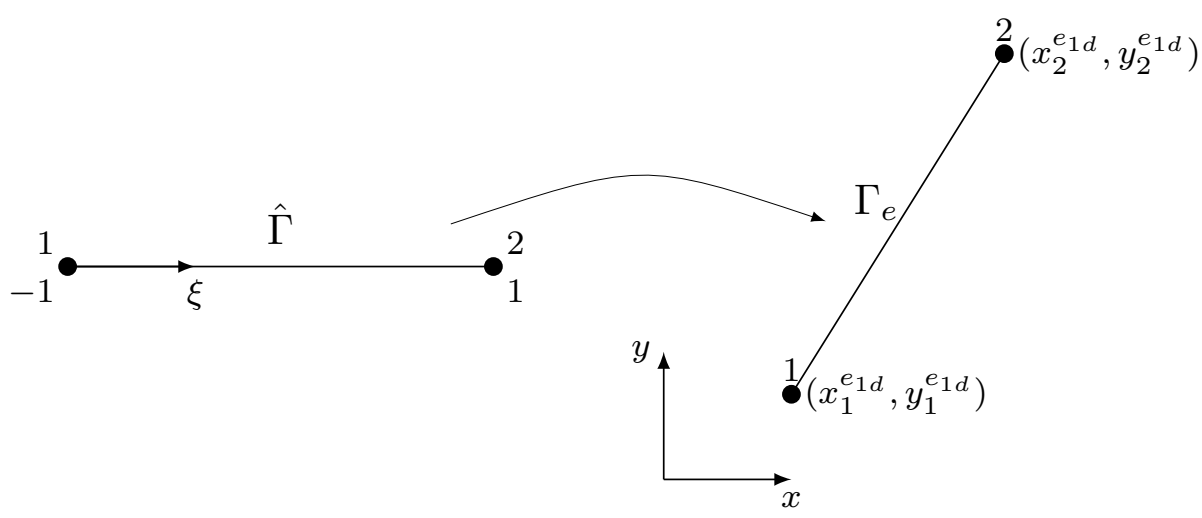

Figure 9: Affine mapping from biunit parent element $\hat{\Gamma}$ to two-node physical element $\Gamma_{e}$.

In (36). $\Delta x^{e_{1 d}}=x_{2}^{e_{1 d}}-x_{1}^{e_{1 d}}$ and $\Delta y^{e_{1 d}}=y_{2}^{e_{1 d}}-y_{1}^{e_{1 d}}$, and the enrichment functions $\boldsymbol{W}$ are

$$
\begin{aligned}
\boldsymbol{W}= & \exp \left[\frac{i k}{2}\left\{\sum_{a=1}^{2} x_{a}^{e_{1 d}} \cos \left(\frac{2 \pi}{q} \boldsymbol{n}\right)+\sum_{a=1}^{2} y_{a}^{e_{1 d}} \sin \left(\frac{2 \pi}{q} \boldsymbol{n}\right)\right\}\right] \\
& \exp \left[\frac{i k \xi}{2}\left\{\Delta x^{e_{1 d}} \cos \left(\frac{2 \pi}{q} \boldsymbol{n}\right)+\Delta y^{e_{1 d}} \sin \left(\frac{2 \pi}{q} \boldsymbol{n}\right)\right\}\right] .
\end{aligned}
$$

The element dependent constant exponent appearing in (37) is

$$
\gamma_{n}^{e_{1 d}}=\frac{1}{2}\left\{\sum_{a=1}^{2} x_{a}^{e_{1 d}} \cos \left(\frac{2 \pi}{q} n\right)+\sum_{a=1}^{2} y_{a}^{e_{1 d}} \sin \left(\frac{2 \pi}{q} n\right)\right\} .
$$


Now, we define the local wave number (a scalar) $k_{n}^{e_{1 d}}=\frac{k}{2}\left\{\Delta x^{e_{1 d}} \cos \left(\frac{2 \pi}{q} n\right)+\Delta y^{e_{1 d}} \sin \left(\frac{2 \pi}{q} n\right)\right\}$ to rewrite (37) for the enrichment direction $n$ as

$$
W_{n}^{e_{1 d}}=\exp \left(i k \gamma_{n}^{e_{1 d}}\right) \exp \left(i k_{n}^{e_{1 d}} \xi\right)
$$

for $n=0, \ldots,(q-1)$. As in case of $\Omega_{\Delta}$, the local wave numbers $k_{n}^{e_{1 d}}$ are dependent on the nodal coordinates of $\Gamma_{e}$. Thus, one needs to use the recursive scheme for all the elements on the boundary. The relevant closed-form expressions for the line integrals on $\hat{\Gamma}$ are presented in Appendix $C$.

Expressing the enrichments $\boldsymbol{W}$ in parent coordinate system reveals the explicit nature of the integrals that appear in (26). These integrals take the form of (29). In the present work, we consider linearly complete FE basis functions for which $m=0,1,2$ and $n=0,1,2$ in (29); use of higher-order finite elements would increase the maximum values that $m$ and $n$ can assume.

\section{Numerical results}

First, to establish the accuracy of the new integration scheme, we compute oscillatory integrals over the biunit square, reference unit triangle, as well as simple convex and non-convex polygons. Then, we demonstrate its capabilities in the PUFEM by solving three benchmark Helmholtz problems, which have been previously considered in the literature.

\subsection{Exact integration of oscillatory functions over polygons}

As an illustrative example, we compute the oscillatory integral

$$
\int_{P} x^{2} y^{3} \exp \left[i k\left\{x \cos \left(\frac{\pi}{2}\right)+y \sin \left(\frac{\pi}{2}\right)\right\}\right] d x d y
$$

over the polygonal domain $P$. The value of this integral using the new scheme is compared to Gauss cubature and to the exact result obtained using symbolic integration in MATLAB ${ }^{\circledR}$.

\subsubsection{Reference triangular element and biunit square}

We compute the integral in (39) over the reference triangle (see Fig. 8) and the biunit square (see Fig. 7). The results for different values of $k$ are listed in Table 1 and Table 2 . For both domains, the new method delivers results that match the exact value of the integral.

Table 1: Numerical computation of the integral in 39 over the reference triangle.

\begin{tabular}{|c|c|c|c|}
\hline$k$ & Gauss cubature & Exact & New method \\
\hline 10 & $(1.30-4.41 i) \times 10^{-4}$ & $(1.30-4.41 i) \times 10^{-4}$ & $(1.30-4.41 i) \times 10^{-4}$ \\
$10^{2}$ & $(3.82-1.04 i) \times 10^{-8}$ & $(3.82-1.04 i) \times 10^{-8}$ & $(3.82-1.04 i) \times 10^{-8}$ \\
$10^{3}$ & $(1.16+30.02 i) \times 10^{-7}$ & $(3.10+1.64 i) \times 10^{-12}$ & $(3.10+1.64 i) \times 10^{-12}$ \\
$10^{4}$ & $(-1.67+1.04 i) \times 10^{-5}$ & $(96.42-6.16 i) \times 10^{-17}$ & $(96.42-6.16 i) \times 10^{-17}$ \\
\hline
\end{tabular}


Table 2: Numerical computation of the integral in 39 , over the biunit square.

\begin{tabular}{|c|c|c|c|}
\hline$k$ & Gauss cubature & Exact & New method \\
\hline 10 & $8.38 i \times 10^{-2}$ & $8.38 i \times 10^{-2}$ & $8.38 i \times 10^{-2}$ \\
$10^{2}$ & $-1.17 i \times 10^{-2}$ & $-1.17 i \times 10^{-2}$ & $-1.17 i \times 10^{-2}$ \\
$10^{3}$ & $-5.08 \times 10^{-6}+7.33 \times 10^{-3} i$ & $-7.47 i \times 10^{-4}$ & $-7.47 i \times 10^{-4}$ \\
$10^{4}$ & $(39.63+9.86 i) \times 10^{-3}$ & $1.27 i \times 10^{-4}$ & $1.27 i \times 10^{-4}$ \\
\hline
\end{tabular}

\subsubsection{Simple polygons}

We now compute the integral in (39) over simple polygons (Fig. 10). The coordinates of the vertices of the polygons are presented in Table 4. Numerical results for the value of the integral over the polygons are listed in Table 3. The results obtained with the new method matches the exact value, thereby establishing its accuracy for any convex or non-convex simple polygon.

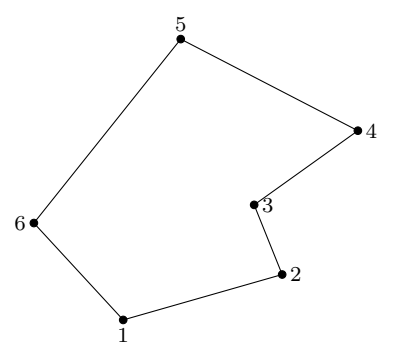

(a)

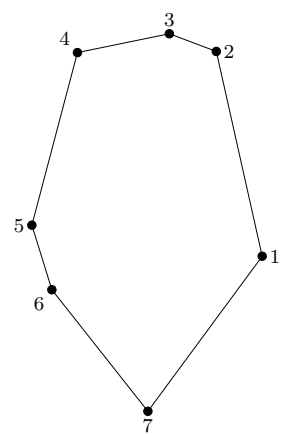

(b)

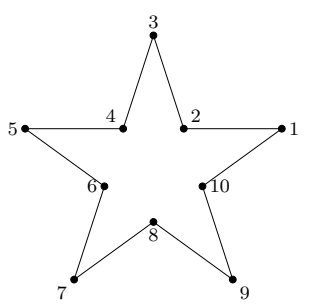

(c)

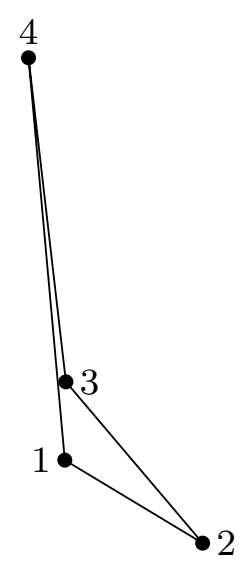

(d)

Figure 10: Simple polygons.

Table 3: Numerical computation of the integral in 39, over the polygonal domains shown in Fig. 10

\begin{tabular}{|c|c|c|c|c|}
\hline \multirow{2}{*}{ Polygon } & \multicolumn{2}{|c|}{$k=10$} & \multicolumn{2}{|c|}{$k=10^{2}$} \\
\hline & Exact & New method & Exact & New method \\
\hline Fig. $10 \mathrm{a}$ & $-14.64-2.2 .68 i$ & $-14.64-2.2 .68 i$ & $(-3.49-3.15 i) \times 10^{-1}$ & $(-3.49-3.15 i) \times 10^{-1}$ \\
\hline Fig. $10 \mathrm{~b}$ & $-19.52+23.00 i$ & $-19.52+23.00 i$ & $(-1.84-1.61 i) \times 10^{-1}$ & $(-1.84-1.61 i) \times 10^{-1}$ \\
\hline Fig. $10 \mathrm{c}$ & $(-36.25+5.72 i) \times 10^{-4}$ & $(-36.25+5.72 i) \times 10^{-4}$ & $(-2.97-13.14 i) \times 10^{-5}$ & $(-2.97-13.14 i) \times 10^{-5}$ \\
\hline Fig. $10 \mathrm{~d}$ & $-0.70+0.42 i$ & $-0.70+0.42 i$ & $(-52.63+1.09 i) \times 10^{-4}$ & $(-52.63+1.09 i) \times 10^{-4}$ \\
\hline
\end{tabular}




\begin{tabular}{|c|c|c|}
\hline Vertex & $x$ & $y$ \\
\hline 1 & 1.900 & 1.000 \\
2 & 4.000 & 1.600 \\
3 & 3.630 & 2.520 \\
4 & 5.000 & 3.500 \\
5 & 2.660 & 4.710 \\
6 & 0.720 & 2.280 \\
\hline
\end{tabular}

(a)

\begin{tabular}{|c|c|c|}
\hline Vertex & $x$ & $y$ \\
\hline 1 & 0.951 & 0.309 \\
2 & 0.225 & 0.309 \\
3 & 1.000 & 0.000 \\
4 & -0.225 & 0.309 \\
5 & -0.951 & 0.309 \\
6 & -0.363 & -0.118 \\
7 & -0.588 & -0.809 \\
8 & 0.000 & -0.382 \\
9 & 0.588 & -0.809 \\
10 & 0.363 & -0.118 \\
\hline
\end{tabular}

(c)

\begin{tabular}{|c|c|c|}
\hline Vertex & $x$ & $y$ \\
\hline 1 & 1.220 & -0.827 \\
2 & 0.132 & 4.027 \\
3 & -3.310 & 4.447 \\
4 & -3.160 & 4.000 \\
5 & -4.240 & -0.091 \\
6 & -3.766 & -1.622 \\
7 & -1.490 & -4.503 \\
\hline
\end{tabular}

(b)

\begin{tabular}{|c|c|c|}
\hline Vertex & $x$ & $y$ \\
\hline 1 & -2.740 & -1.888 \\
2 & -0.643 & -3.151 \\
3 & -2.723 & -0.697 \\
4 & -3.292 & 4.233 \\
\hline
\end{tabular}

(d)

Table 4: Coordinates of the vertices of the polygons in Fig. 10 .

\subsubsection{Timings}

The computational cost of the proposed scheme is independent of $k$. To establish this claim, we compute

$$
\mathcal{I}_{[m, n]}=\int_{\hat{\Omega}} x^{m} y^{n} \exp \left(i \boldsymbol{k}_{n} \cdot \boldsymbol{x}\right) d \boldsymbol{x}
$$

for different values of $m, n$, and $k$ and plot the elapsed wall-clock time $\Delta t$ versus $k$ in Fig. 11 . This is performed for $\hat{\Omega}=\Omega_{\triangle}$ as well as $\hat{\Omega}=\Omega_{\square}$. We observe that the computational time is almost the same for any $k$. Also, an increase in the value of either $m$ or $n$ implies more number of executions of the recursive scheme, which increases $\Delta t$. 


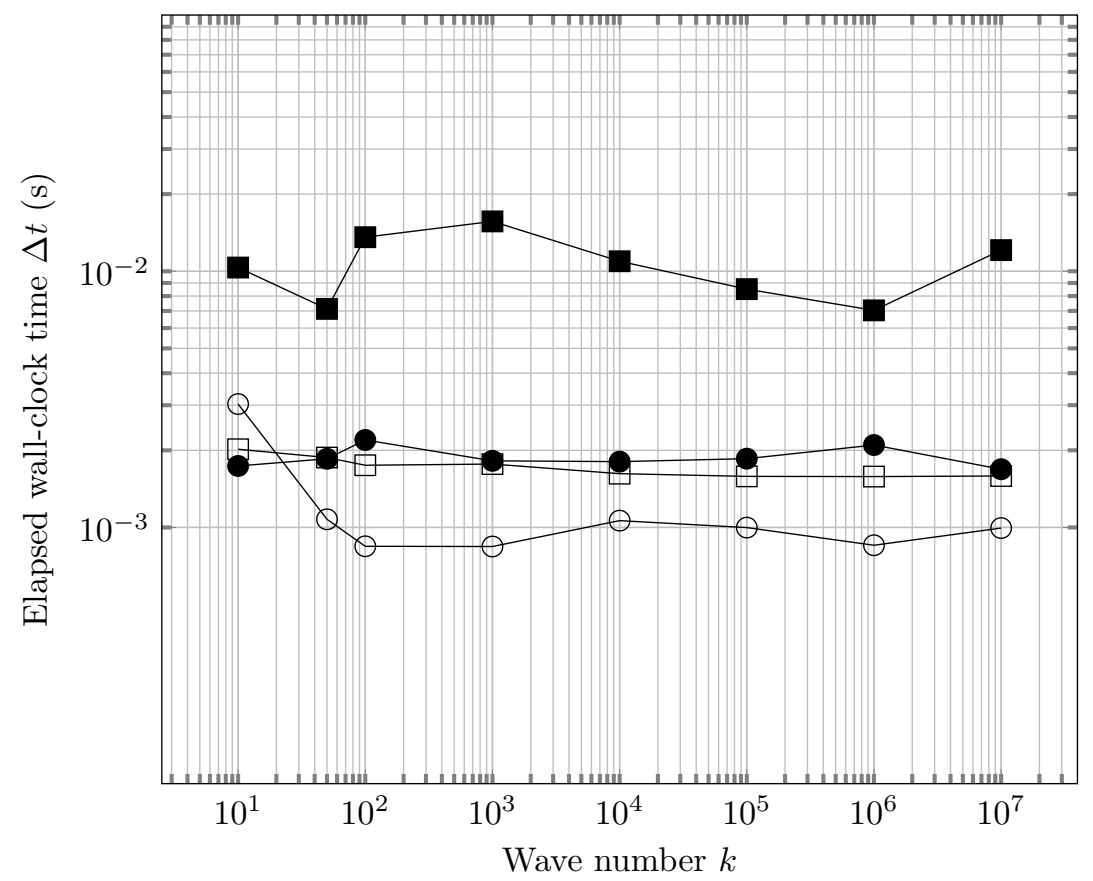

$-m=2, n=0$
$-m=0, n=1$
$\square-m=2, n=1$
$\square m=0, n=2$

(a)

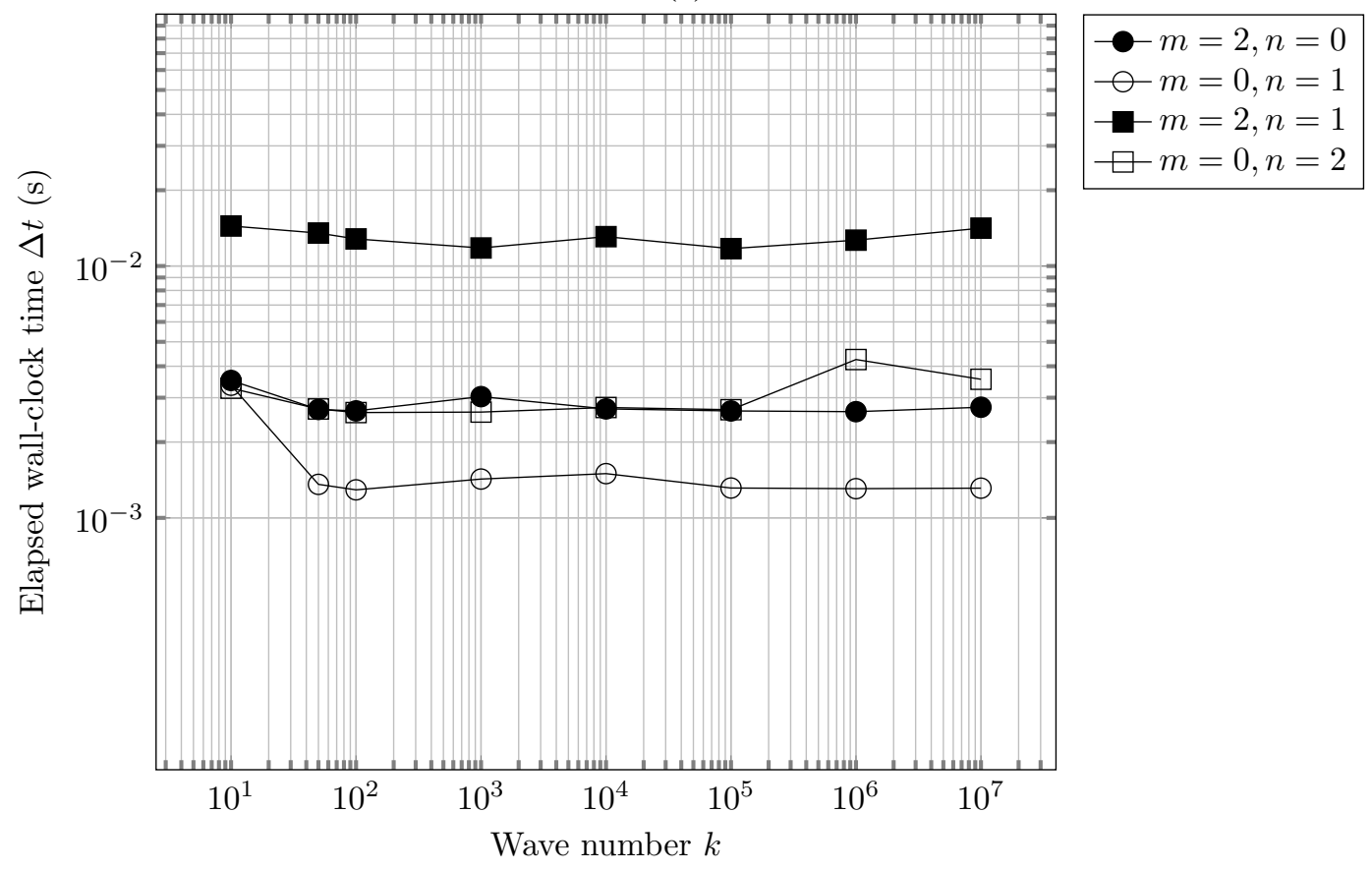

(b)

Figure 11: Elapsed wall-clock time $\Delta t$ versus $k$ for different $m$ and $n$ in 40). (a) Reference triangle; and (b) Biunit square.

\subsection{Planewave scattered in a square domain}

Referring to Fig. 4, we consider the problem domain $\Omega=(-1,1)^{2}$ (see Fig. 12b with the scatter $\Omega_{s}$ being absent (void), and $\partial \Omega_{t}$ being the boundary of the domain. We seek the numerical 
solution of the following Helmholtz problem:

$$
\begin{array}{ll}
-\nabla^{2} u(\boldsymbol{x})-k^{2} u(\boldsymbol{x})=0 & \text { in } \Omega \\
\frac{\partial u(\boldsymbol{x})}{\partial n}-i k u(\boldsymbol{x})=g(\boldsymbol{x}) & \text { on } \partial \Omega_{t},
\end{array}
$$

where we choose the function $g(\boldsymbol{x})$ on $\partial \Omega_{t}$ such that the exact solution $u(\boldsymbol{x})=\exp (i k \boldsymbol{p} \cdot \boldsymbol{x})$ represents a planewave traveling in the direction $\boldsymbol{p}=\cos \Theta \boldsymbol{e}_{1}+\sin \Theta \boldsymbol{e}_{2}$, This results in

$$
g(\boldsymbol{x})=i k \exp (i k \boldsymbol{p} \cdot \boldsymbol{x}) \begin{cases}(\cos \Theta-1), & \text { on } x=1 \\ (\sin \Theta-1), & \text { on } y=1 \\ (-\cos \Theta-1), & \text { on } x=-1 \\ (-\sin \Theta-1), & \text { on } y=-1 .\end{cases}
$$

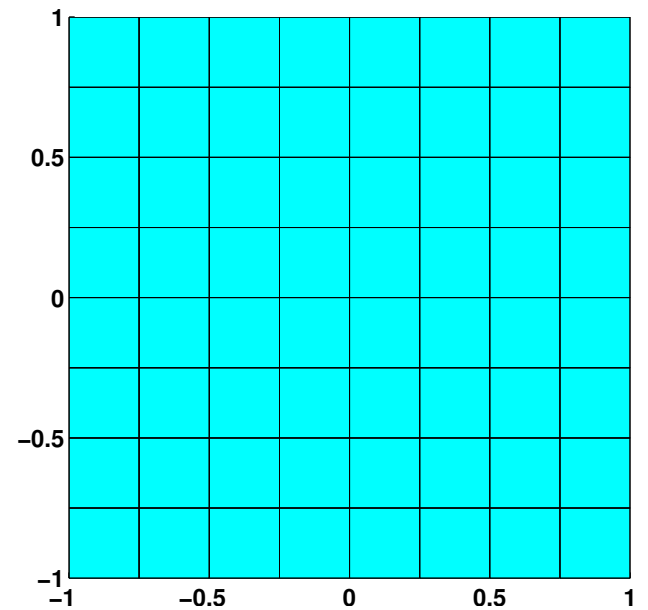

(a)

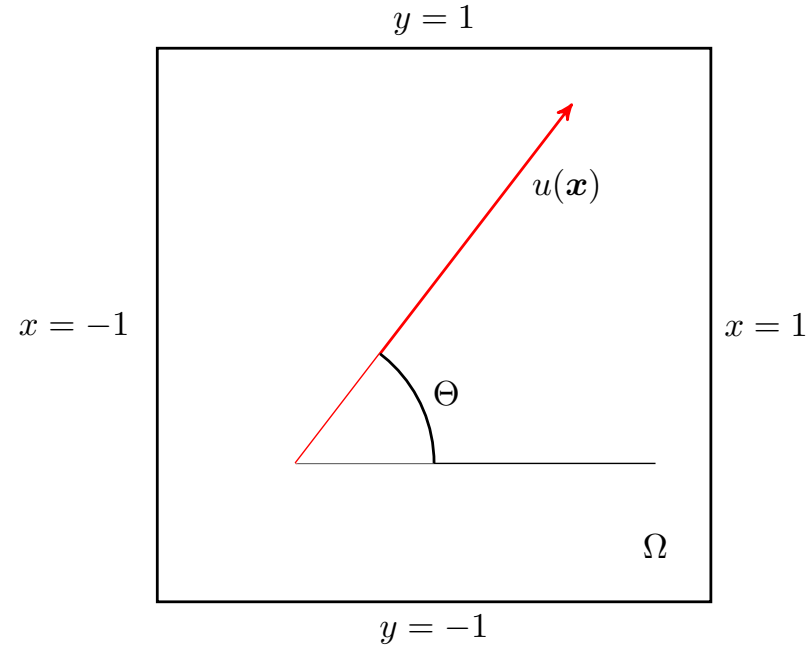

(b)

Figure 12: Planewave scattered in a square domain: (a) Finite element mesh of four node rectangular elements; and (b) Problem domain $\Omega$. The red arrow represents the direction of travel of the exact solution $u(\boldsymbol{x})$, which is a planewave traveling along the angle $\Theta$ with respect to the horizontal direction.

\subsubsection{Enrichment direction aligned with $\boldsymbol{p}$}

In the first numerical experiment, we let $\boldsymbol{p}=\cos (\pi / 2) \boldsymbol{e}_{1}+\sin (\pi / 2) \boldsymbol{e}_{2}(\Theta=\pi / 2)$ with the exact solution

$$
u(\boldsymbol{x})=\exp (i k y)
$$

We conduct PUFE computations over uniform Cartesian meshes of the type shown in Fig. 12a. To assess the error, the relative $L^{2}$-norm and the relative $H^{1}$-seminorm of the approximation error are used, which are defined as

$$
\|e\|_{L^{2}}=\frac{\left\|u-u^{h}\right\|_{L^{2}}}{\|u\|_{L^{2}}}, \quad|e|_{H^{1}}=\frac{\left\|\nabla\left(u-u^{h}\right)\right\|_{L^{2}}}{\|\nabla u\|_{L^{2}}} .
$$


Four $(q=4)$ and eight $(q=8)$ equispaced planewave directions in [0,2 $\pi]$ are used (see Fig. 13) on a uniform mesh with grid-spacing $h=0.5$ (25 nodes). For both $q=4$ and 8 , we are assured that at least one enrichment function is aligned in the direction $\boldsymbol{p}$, and hence the PUFE solution matches the exact solution for $k=40$ and $k=120$ (see Table 5). As an additional test, we choose $k=120$ and $\Theta=0$, with a single enrichment function along $\Theta=0$. For $h=0.5$ (50 nodes), the relative errors in the PUFE solution are $\|e\|_{L^{2}}=1.8 \times 10^{-16}$ and $|e|_{H^{1}}=1.8 \times 10^{-16}$.

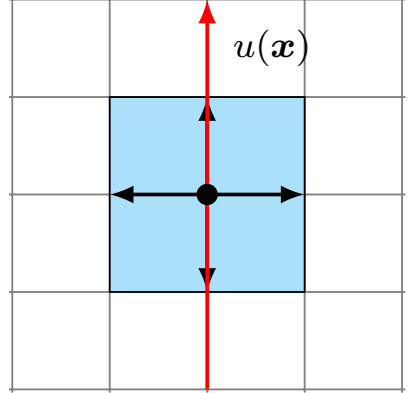

(a)

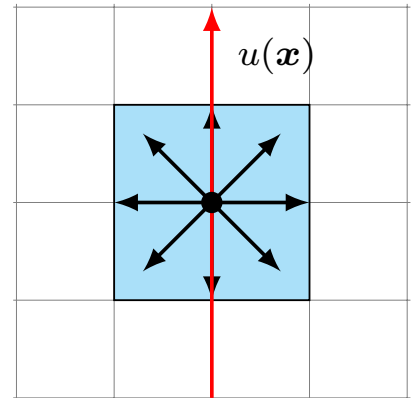

(b)

Figure 13: Enrichment direction coincident with $\boldsymbol{p}=\cos (\pi / 2) \boldsymbol{e}_{1}+\sin (\pi / 2) \boldsymbol{e}_{2}$. The red arrow indicates the direction of travel of $u(\boldsymbol{x})$ and the black arrows indicate the direction of the enrichment functions associated with the node marked by the filled black circle. The cases (a) $q=4$ and (b) $q=8$ are considered.

Table 5: Relative error norms for the test problem with enrichment direction aligned with $\boldsymbol{p}$.

\begin{tabular}{|c|c|c|c|c|}
\hline \multirow{2}{*}{$q$} & \multicolumn{2}{|c|}{$k=40$} & \multicolumn{2}{|c|}{$k=120$} \\
\hline & $\|e\|_{L^{2}}$ & $|e|_{H^{1}}$ & $\|e\|_{L^{2}}$ & $|e|_{H^{1}}$ \\
\hline 4 & $3.1 \times 10^{-15}$ & $3.1 \times 10^{-15}$ & $9.9 \times 10^{-15}$ & $1.0 \times 10^{-14}$ \\
\hline 8 & $3.7 \times 10^{-15}$ & $3.7 \times 10^{-15}$ & $1.0 \times 10^{-14}$ & $1.0 \times 10^{-14}$ \\
\hline
\end{tabular}

\subsubsection{Enrichment direction not aligned with $\boldsymbol{p}$}

For this case, we choose $\Theta=1.1(\pi / 2)=99^{\circ}$ so that the exact solution is

$$
u(\boldsymbol{x})=\exp (i k(x \cos \Theta+y \sin \Theta)
$$

The PUFE solution of (41) is computed for $q=4,8$, and 12 . The cases $k=40$ and 120 are considered. Referring to Fig. 14, note that none of the enrichment functions (black arrows) are aligned with the exact solution (red arrow) $u(\boldsymbol{x})$. In Fig. 15, we show plots of the relative error norms versus the square root of the total number of degrees of freedom. For a given $q$, we observe monotonic convergence (once pollution effects die out). Additionally, we report the 1-norm condition number estimate, $\operatorname{cond}_{1}(\boldsymbol{K})$, of the global stiffness matrix $\boldsymbol{K}$ using condest routine of MATLAB ${ }^{\circledR}$. With increase in $q$, there is a sharp increase in the condition number (see Fig. 16), which points to the well-known issue of ill-conditioning of $\boldsymbol{K}$ in the PUFEM. The real and imaginary parts of a typical PUFE solution with $q=10$ for $k=40$ and $\Theta=\frac{\pi}{3}$ are presented in Fig. 17 . 


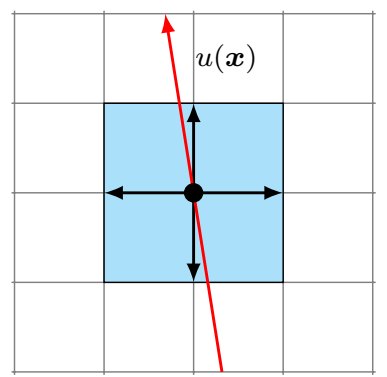

(a)

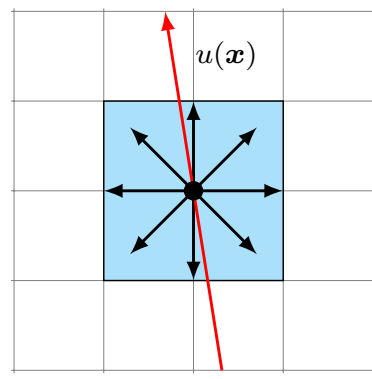

(b)

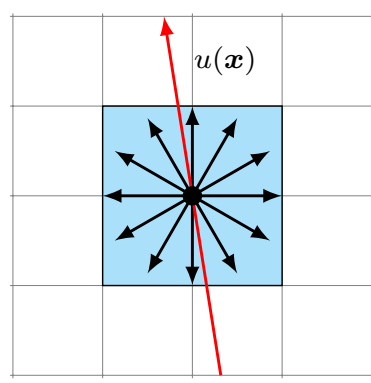

(c)

Figure 14: Enrichment direction not aligned with $\boldsymbol{p}=\cos (1.1 \pi / 2) \boldsymbol{e}_{1}+\sin (1.1 \pi / 2) \boldsymbol{e}_{2}$. The red arrow indicates the direction of travel of $u(x)$ and the black arrows indicate the direction of the enrichment functions associated with the node marked by the filled black circle. The cases (a) $q=4$, (b) $q=8$ and (c) $q=12$ are considered.

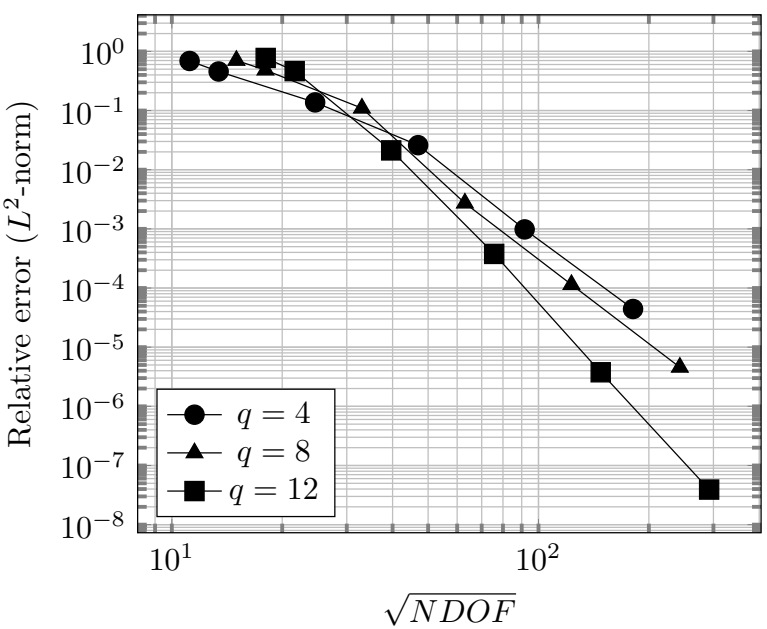

(a)

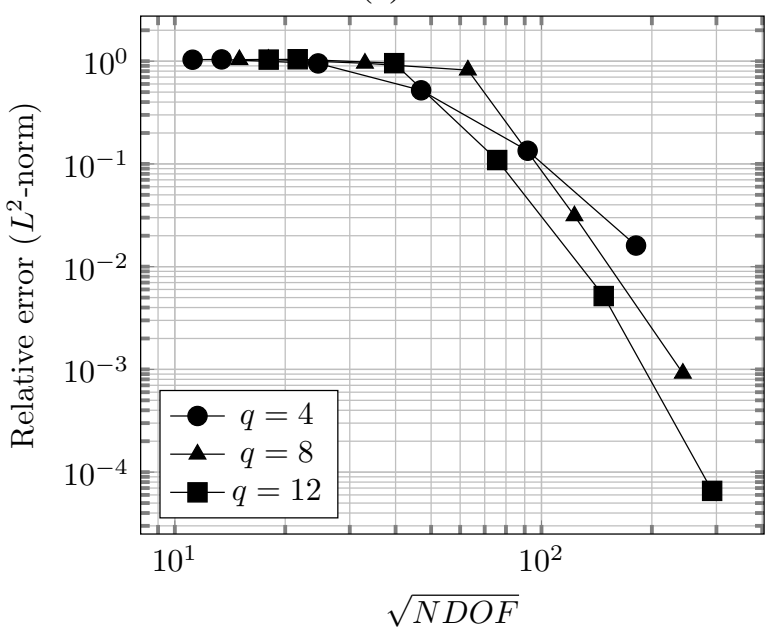

(c)

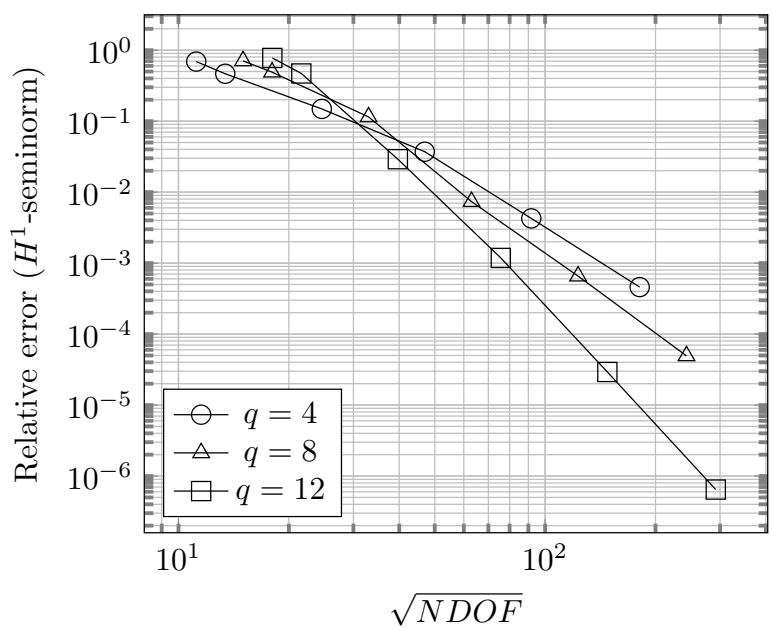

(b)

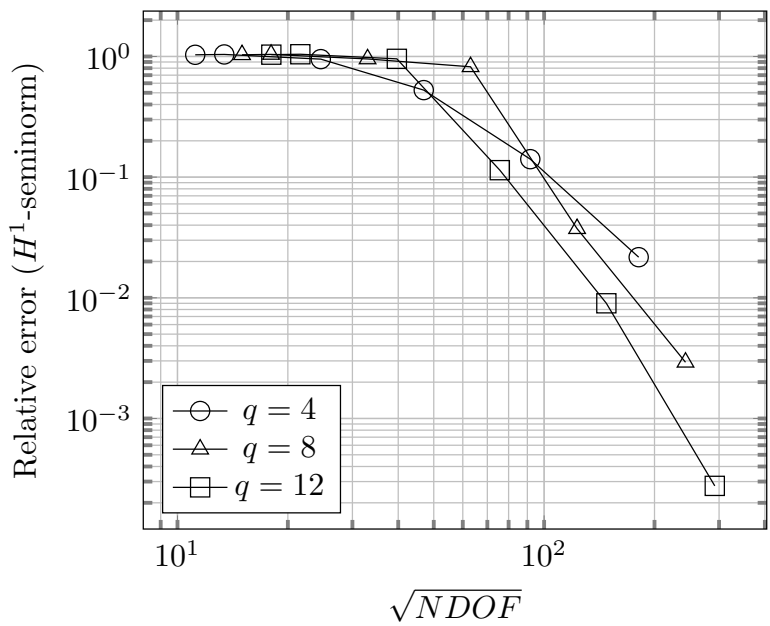

(d)

Figure 15: Relative error norms for the test problem with the enrichment direction not aligned with $\boldsymbol{p}$. (a), (b) $k=40$; and (c), (d) $k=120$. 


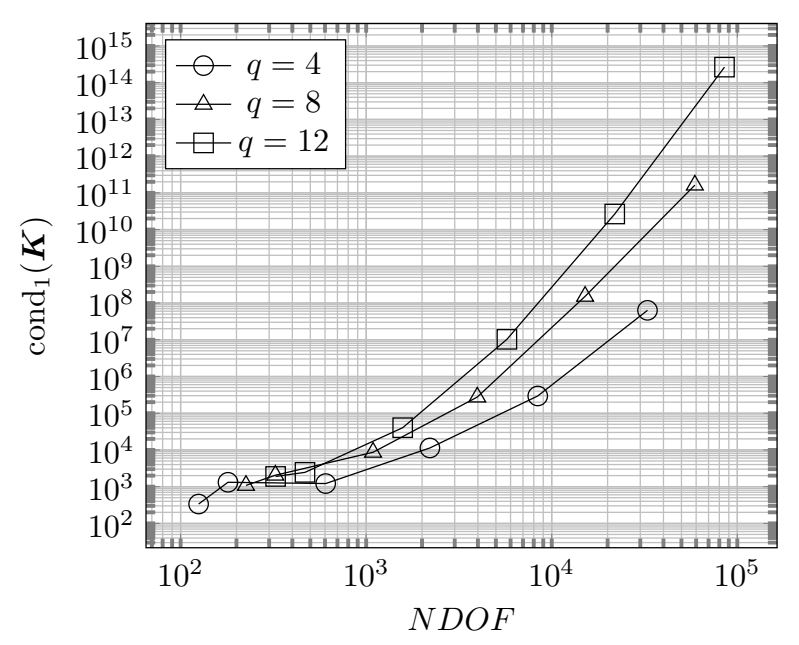

(a)

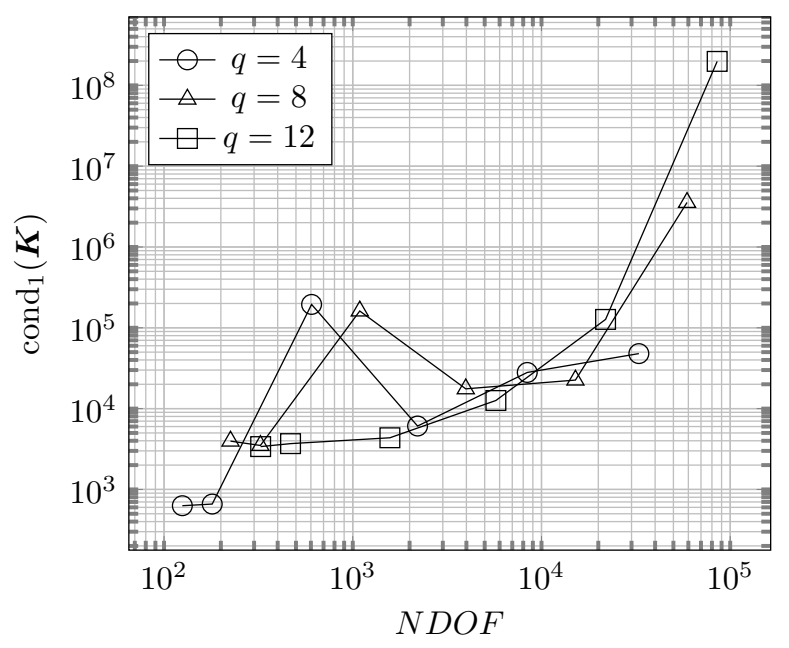

(b)

Figure 16: Estimate of the condition number of $\boldsymbol{K}$ for (a) $k=40$ and (b) $k=120$.

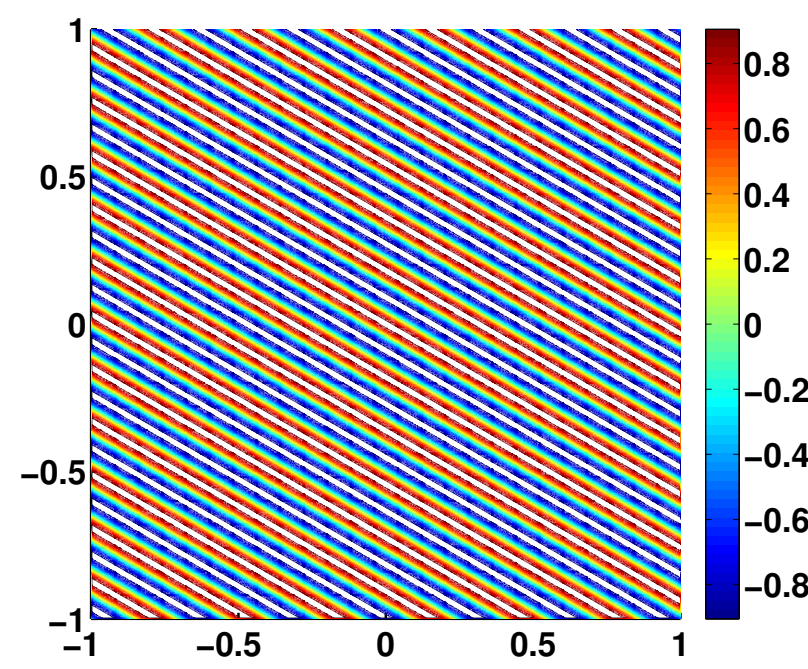

(a)

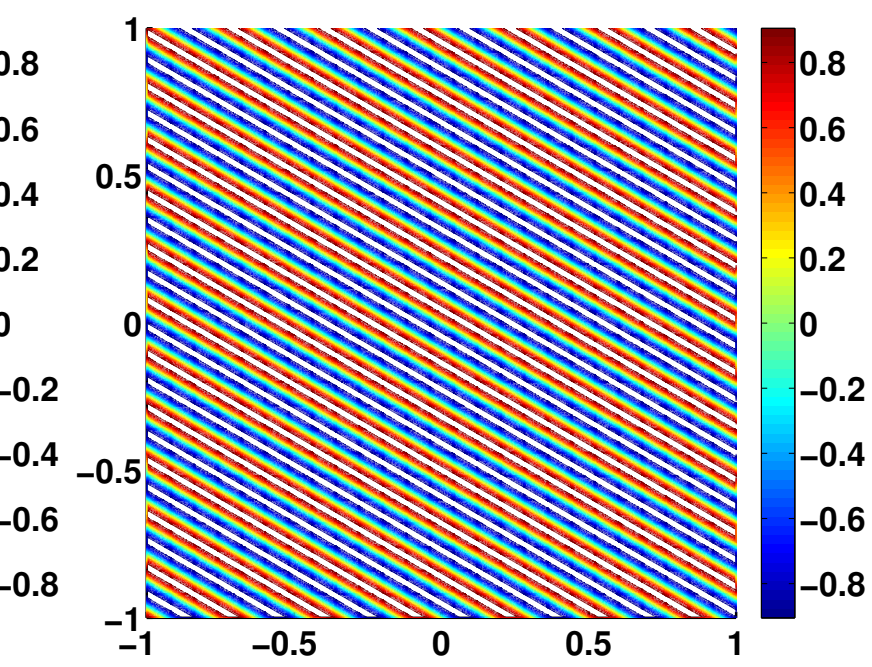

(b)

Figure 17: PUFE solution for $k=40, q=10$ and $\Theta=\pi / 3$. (a) Real component; and (b) Imaginary component.

\subsection{Planewave scattered by circular cylinder}

We study the exterior Helmholtz problem corresponding to harmonic planewave $u_{I}$ scattered by sound hard bounded cylindrical obstacle $\Omega_{s}$. The scattered wave is $u_{S}$, and the domain $\Omega_{s}$ is bounded by $\partial \Omega_{s}$ of radius $R_{1}$. We assume $u_{I}$ is traveling along the direction $\boldsymbol{p}_{I}$, and is incident on the cylinder at an angle $\theta_{0}$ with respect to the horizontal direction. In polar coordinates,

$$
u_{I}(r, \theta)=U_{0} \exp \left(i k r \cos \left(\theta-\theta_{0}\right)\right),
$$

where $U_{0}$ is the magnitude and $k$ is the wave number. For brevity and without loss of generality, we assume $\theta_{0}=0$, that is $\boldsymbol{p}_{I}=\boldsymbol{e}_{1}$ such that

$$
u_{I}(r, \theta)=U_{0} \exp (i k r \cos (\theta))=U_{0} \exp \left(i k \boldsymbol{p}_{I} \cdot \boldsymbol{x}\right) .
$$


Additionally, we truncate the infinite extent of the exterior problem by introducing a truncation boundary $\partial \Omega_{t}$ at some finite radius $R_{2}$. The annular domain enclosed by the boundaries $\partial \Omega_{s}$ and $\partial \Omega_{t}$ is now our problem domain $\Omega$ (see Fig. 4). Lastly, we denote $v$ as the outward normal vector to $\partial \Omega=\partial \Omega_{t} \cup \partial \Omega_{s}$.

The exact solution for the scattered wave (by sound hard cylinder) in polar coordinates is [3]:

$$
u(r, \theta)=-U_{0} \sum_{n=0}^{\infty} \epsilon_{n} i^{n} \frac{J_{n}^{\prime}\left(k R_{1}\right)}{H_{n}^{\prime(1)}\left(k R_{1}\right)} H_{n}^{(1)}(k r) \cos (n \theta) .
$$

In (45), the Jacobi symbol $\epsilon_{n}$ is defined as

$$
\epsilon_{n}=\left\{\begin{array}{ll}
1 & n=0 \\
2 & n \neq 0
\end{array},\right.
$$

$H_{n}^{(1)}(\cdot)$ is the cylindrical Hankel function of the first kind, and $J_{n}(\cdot)$ is the cylindrical Bessel function of the first kind.

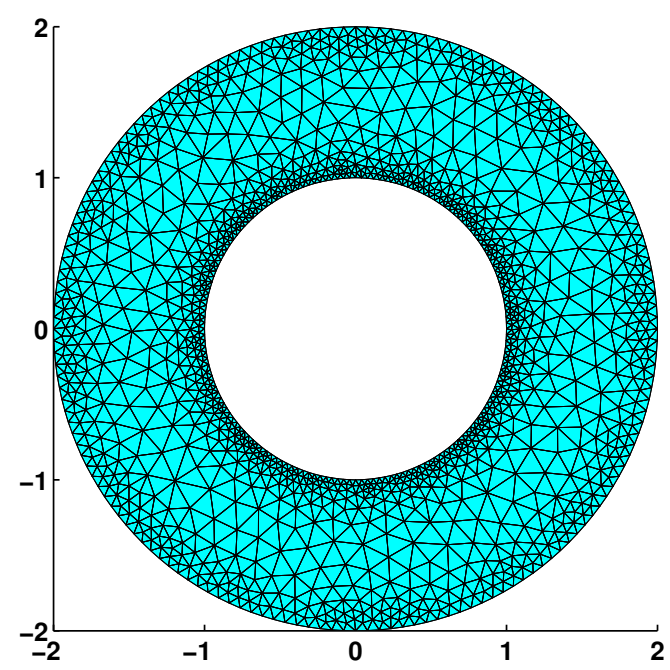

(a)

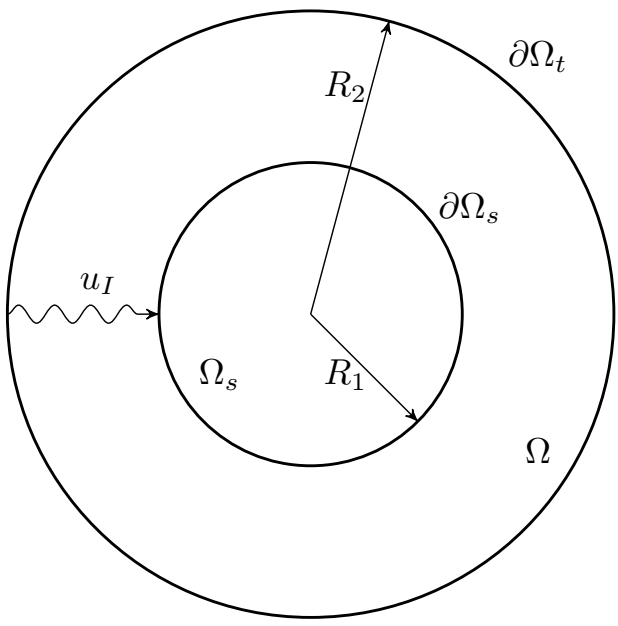

(b)

Figure 18: Planewave scattered by circular cylinder. (a) Unstructured finite element mesh of 3-node triangular element with $h_{\max }=0.25$. Number of divisions along $\partial \Omega_{s}$ and $\partial \Omega_{t}$ is set to 160 to accurately represent the curvature of these boundaries; and (b) Annular domain $\Omega$ with inner and outer radii of $R_{1}=1$ and $R_{2}=2$, respectively. The incident wave $u_{I}$ is traveling along the horizontal direction and is scattered by the boundary $\partial \Omega_{s}$ of the bounded obstacle $\Omega_{s}$. The truncation boundary is $\partial \Omega_{t}$.

We now setup the Helmholtz problem associated with the scattered wave $u_{S}(\boldsymbol{x})$. The sound hard obstacle condition on $\partial \Omega_{s}$ results in the following Neumann type boundary condition:

$$
\frac{\partial u_{S}(\boldsymbol{x})}{\partial \boldsymbol{v}}=-\frac{\partial u_{I}(\boldsymbol{x})}{\partial \boldsymbol{v}}=g_{1}(\boldsymbol{x})
$$

Imposing the exact outgoing condition of (21) on $\Omega$ leads to the computationally expensive evaluation of a nonlocal integral operator [3] on $\partial \Omega_{t}$. Various approximate absorbing boundary condition 
(ABC) are used to resolve this issue. Since they are not exact, they in general cause small artificially reflected waves when the scattered wave $u_{S}(\boldsymbol{x})$ bounces off $\partial \Omega_{t}$. Instead, for simplicity, we use the exact solution in 45 to impose a Robin boundary condition on $\partial \Omega_{t}$ :

$$
\frac{\partial u(\boldsymbol{x})}{\partial \boldsymbol{v}}-i k u(\boldsymbol{x})=g_{2}(\boldsymbol{x}) \quad \boldsymbol{x} \in \partial \Omega_{t} .
$$

We consider the Helmholtz problem

$$
\begin{aligned}
-\nabla^{2} u_{S}(\boldsymbol{x})-k^{2} u_{S}(\boldsymbol{x})=0 & \text { in } \Omega \\
\frac{\partial u_{S}(\boldsymbol{x})}{\partial \boldsymbol{v}}=g_{1}(\boldsymbol{x}) & \text { on } \partial \Omega_{s} \\
\frac{\partial u_{S}(\boldsymbol{x})}{\partial \boldsymbol{v}}-i k u_{S}(\boldsymbol{x})=g_{2}(\boldsymbol{x}) & \text { on } \partial \Omega_{t} .
\end{aligned}
$$

The weak form of the Helmholtz problem is used to implement the planewave-enriched PUFEM. In the numerical experiments, we set $U_{0}=1, R_{1}=1$, and $R_{2}=2$ in (45). It is desirable to use relatively large-sized elements that encompass multiple wavelengths, but accurate geometric representation of the boundary is also paramount. Since we use lower-order finite elements, we resort to use of higher mesh-density in the region near $\partial \Omega_{s}$ and $\partial \Omega_{t}$. Comparative studies are carried out with 40, 80, and 160 number of divisions on these boundaries and it is found that 160 divisions is required to ensure accurate results and monotonic convergence. Hereon, we restrict ourselves to such meshes (see Fig. 18a).

We conduct $q$-convergence [19] studies with the PUFE for $q=0$ (FE), ., 10. In Fig. 19. numerical results are shown for $k=40$ and $k=60$. Alongside, we also report $\operatorname{cond}_{1}(\boldsymbol{K})$ for these cases in Fig. 20. The real and imaginary parts of the PUFE solution for $k=120$ with $q=10$ $\left(h_{\max }=0.03\right)$ are presented in Fig. 21. We mention in passing that in Fig. 19b the last data-point (i.e., $q=10$ ) is erroneous because of the high condition number of $\boldsymbol{K}$. This is also apparent from Fig. $20 \mathrm{~b}$

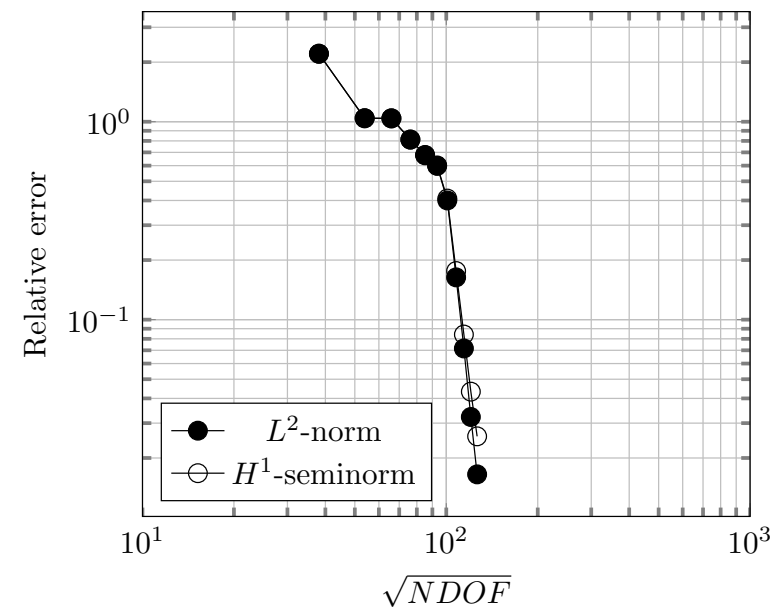

(a)

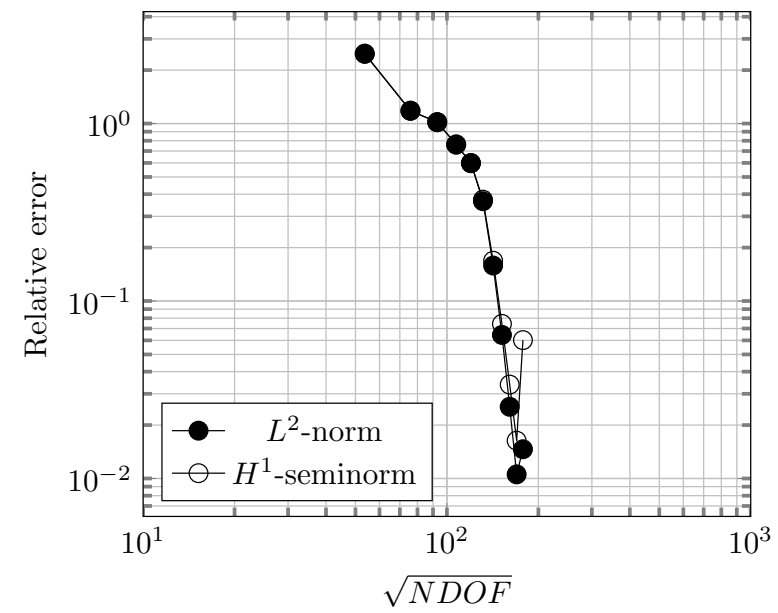

(b)

Figure 19: Relative error norms for the problem of a planewave scattered by a circular cylinder. (a) $k=40$ with $h_{\max }=0.2$; and (b) $k=60$ with $h_{\max }=0.05$. 


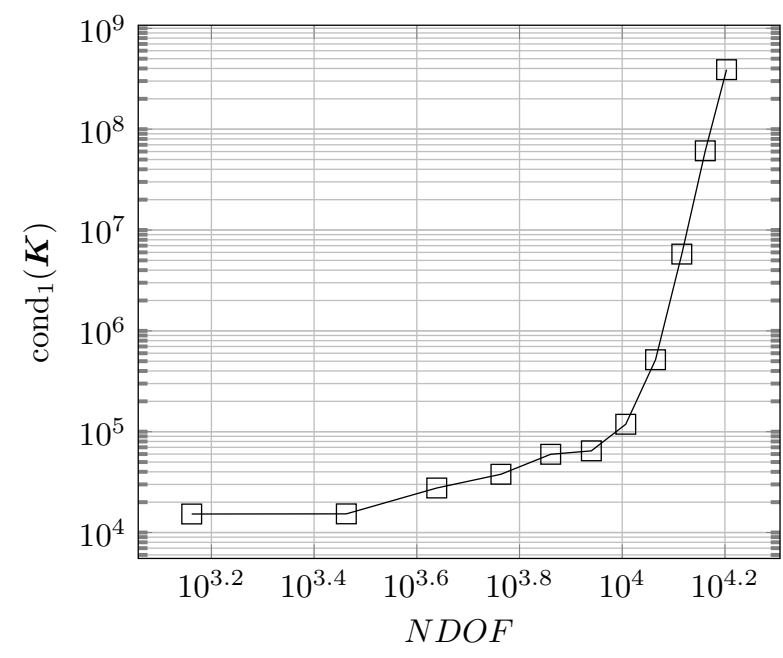

(a)

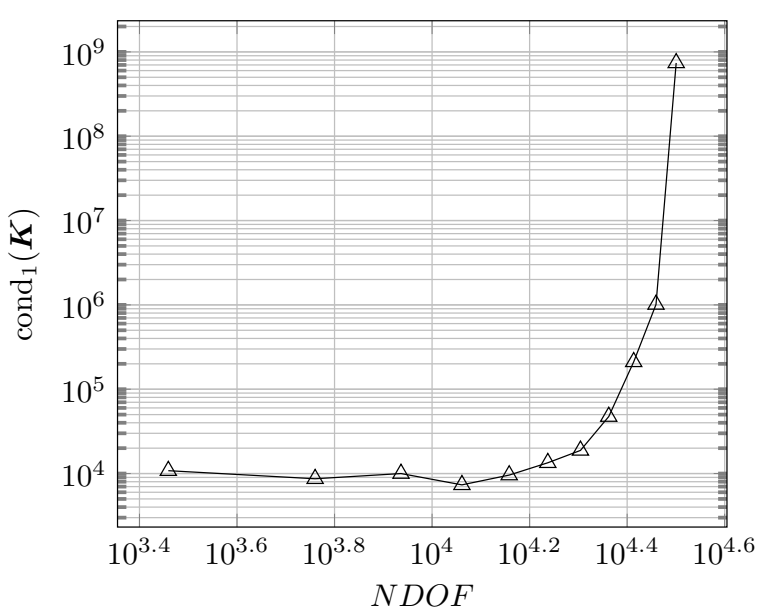

(b)

Figure 20: Estimate of the condition number of $\boldsymbol{K}$ for (a) $k=40$ and (b) $k=60$.

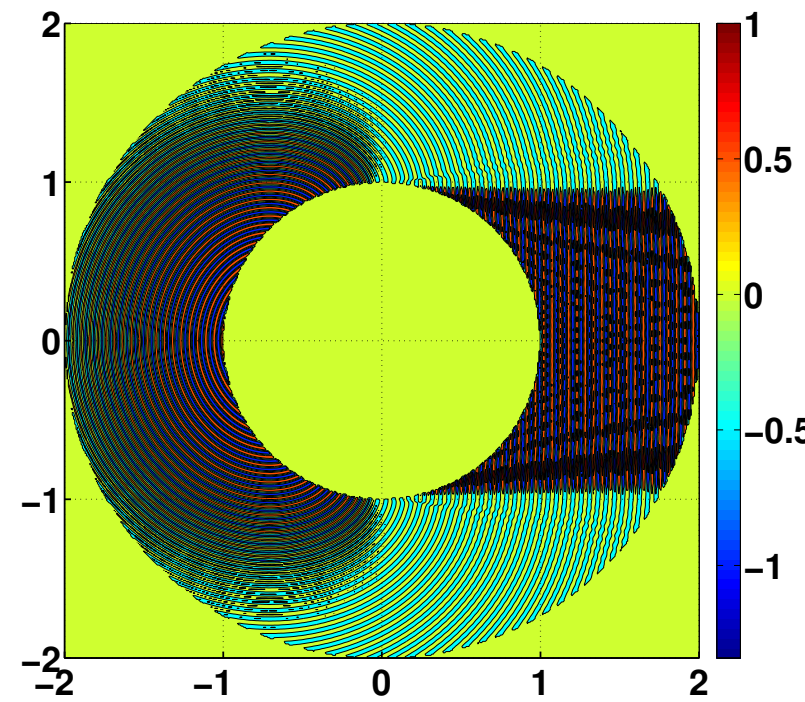

(a)

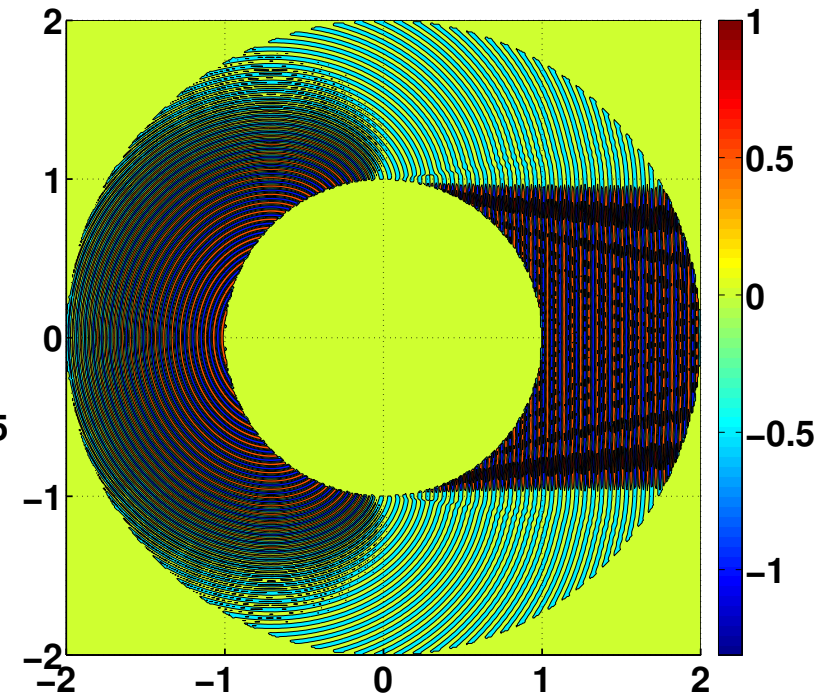

(b)

Figure 21: PUFE solution for the problem of a planewave scattered by a circular cylinder $\left(k=120, q=10, h_{\max }=\right.$ 0.03). (a) Real component; and (b) Imaginary component.

\subsection{Planewave scattered inside a duct with rigid walls}

We consider a benchmark Helmholtz problem that has appeared in the literature of duct acoustics and wave scattering [32-34]. The problem domain $\Omega=(0,2) \times(0,1)$ mimics a longitudinal cross-section of a rigid wall duct. The problem represents uniform flow along the axial direction ( $x$-axis in Fig. 22b) within the duct and the Helmholtz problem corresponds to sound wave propagation in the flowing fluid. In the present work, as in Reference [34], we consider the special case of a stationary fluid. The schematic of the problem domain is shown in Fig. 22b. 


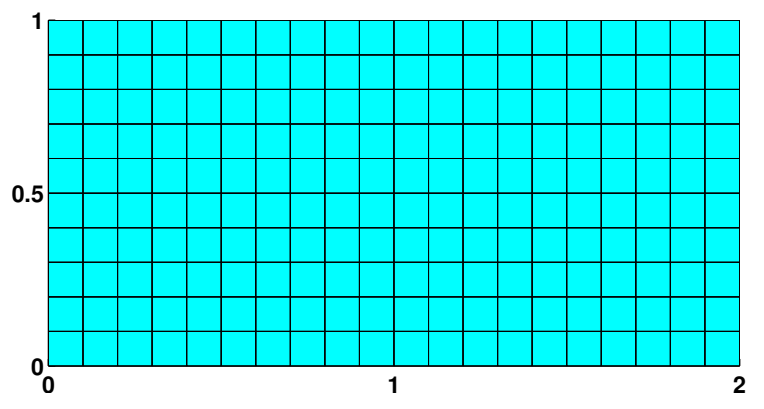

(a)

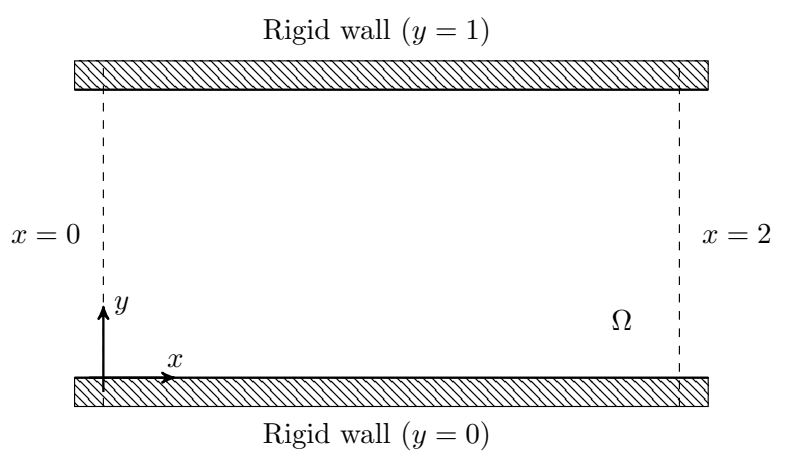

(b)

Figure 22: Planewave scattered inside a duct with rigid walls. (a) Uniform Cartesian mesh of rectangular elements with mesh size $h=0.1$; (b) Problem domain $\Omega=(0,2) \times(0,1)$.

The Helmholtz problem is:

$$
\begin{aligned}
-\nabla^{2} u(\boldsymbol{x})-k^{2} u(\boldsymbol{x})=0 & \text { in } \Omega \\
\frac{\partial u(\boldsymbol{x})}{\partial n}=\cos (m \pi y) & \text { on } x=0 ; \text { mode number } m \in \mathbb{N} \\
\frac{\partial u(\boldsymbol{x})}{\partial n}+i k u=0 & \text { on } x=2 \\
\frac{\partial u(\boldsymbol{x})}{\partial n}=0 & \text { on } y=0 \text { and } y=1 .
\end{aligned}
$$

Physically the condition at the edges: $y=0$ and $y=1$ corresponds to sound hard scatter (rigid wall). Set of non-homogeneous Neumann boundary conditions at: $x=0$ and $x=2$ are also imposed as well. The exact solution of 477 is [32-34]:

$$
u(\boldsymbol{x})=\cos (m \pi y)\left[A_{1} \exp \left(-i \kappa_{x} x\right)+A_{2} \exp \left(i \kappa_{x} x\right)\right]
$$

with $\kappa_{x}=\sqrt{k^{2}-m^{2} \pi^{2}}$, and $A_{1}, A_{2}$ are the solution of

$$
i\left[\begin{array}{cc}
\kappa_{x} & -\kappa_{x} \\
\left(k-\kappa_{x}\right) \exp \left(-2 i \kappa_{x}\right) & \left(k+\kappa_{x}\right) \exp \left(2 i \kappa_{x}\right)
\end{array}\right]\left\{\begin{array}{l}
A_{1} \\
A_{2}
\end{array}\right\}=\left\{\begin{array}{l}
1 \\
0
\end{array}\right\} .
$$

In the numerical discretization, we adopt uniform rectangular meshes. Denoting the cut-off value of mode number $m$ as $m_{\text {cut }}=k / \pi$, we only show numerical results for the case of propagating modes, i.e., $m \leq m_{\text {cut }}$. The case of evanescent modes ( $m>m_{\text {cut }}$ ) is not reported. Specifically, we consider the case of propagating mode with $m=\left\lfloor m_{\text {cut }}\right\rfloor$. As previously shown In Fig. 23, we plot the relative error norms versus the square root of the number of degrees for freedom for $k=40$ and $k=120$. In the PUFE computations, we use $q=4,8$, and 12. Different mesh-resolutions for a given value of $q$ are used to demonstrate monotonic convergence (after pollution effects die out). Additionally, we also report $\operatorname{cond}_{1}(\boldsymbol{K})$ for these cases in Fig. 24. For $k=60$ and using $q=12$, the real and imaginary components of the PUFE solution are presented in Fig. 25 . 


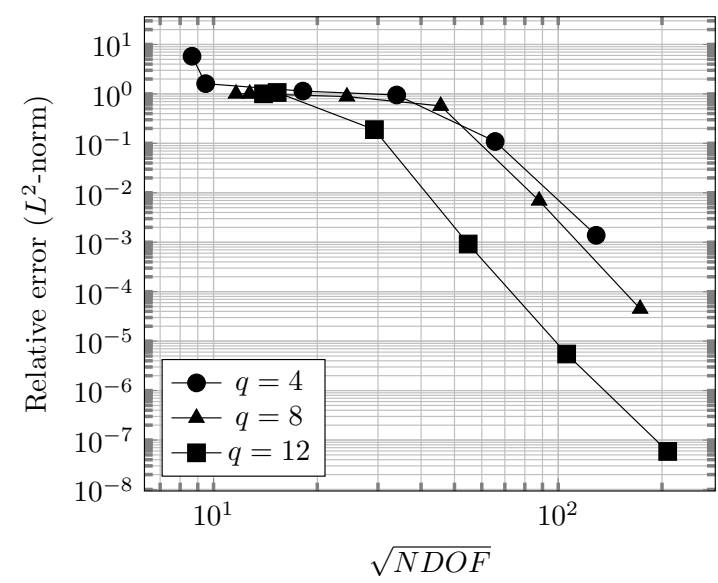

(a)

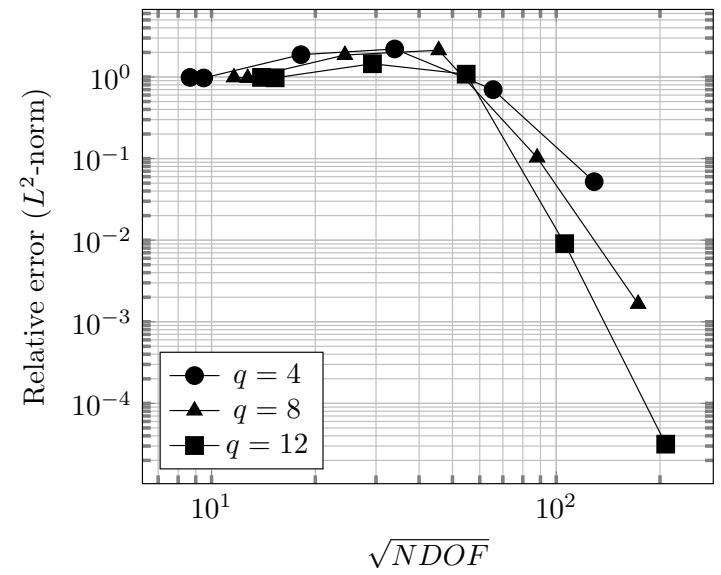

(c)

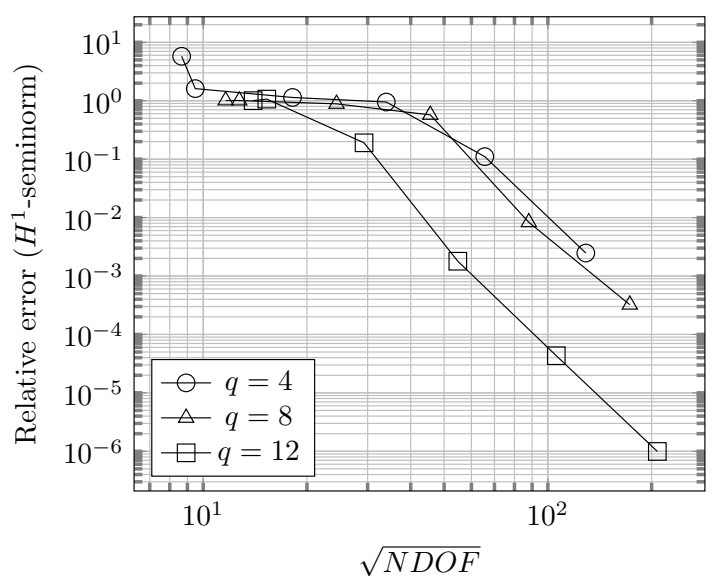

(b)

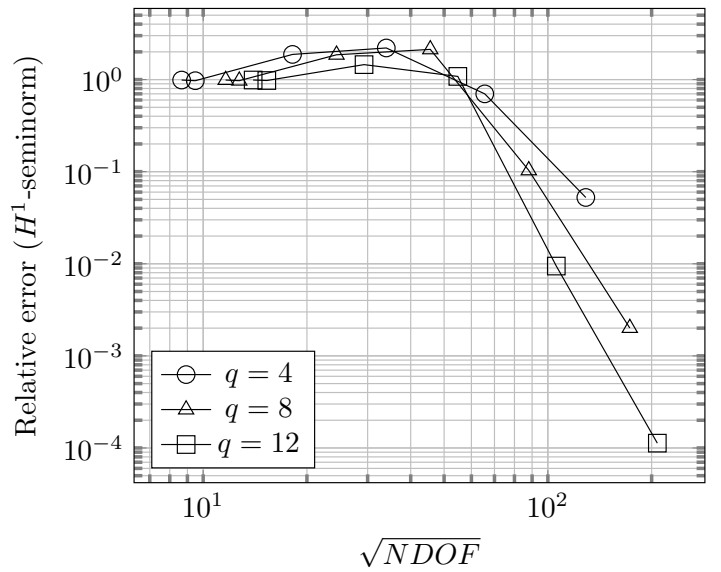

(d)

Figure 23: Relative error norms for the problem of a planewave scattered inside a duct with rigid walls. (a), (b) $k=40$; and (c), (d) $k=120$.

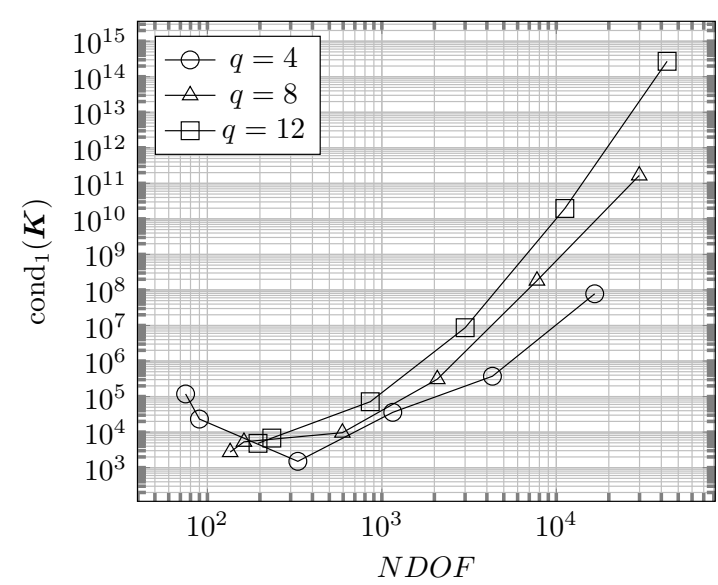

(a)

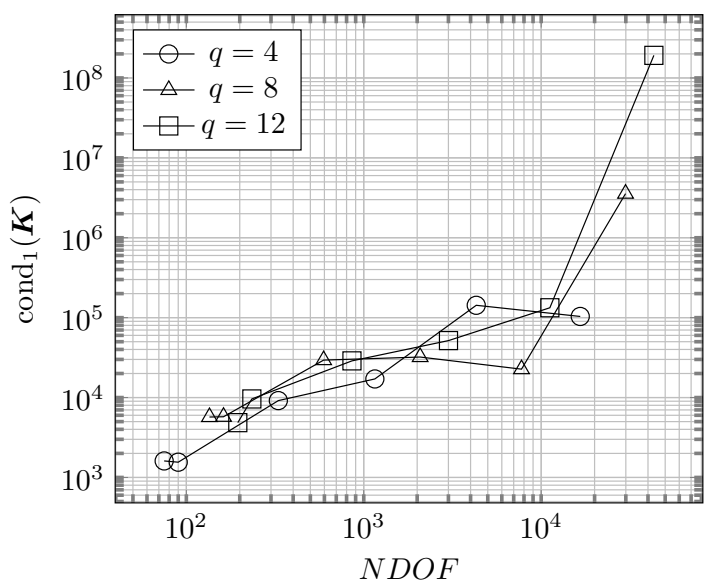

(b)

Figure 24: Estimate of the condition number of $\boldsymbol{K}$ for (a) $k=40$ and (b) $k=120$. 


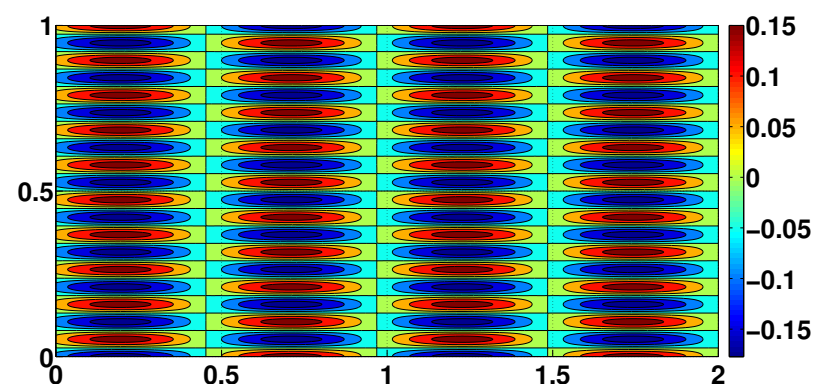

(a)

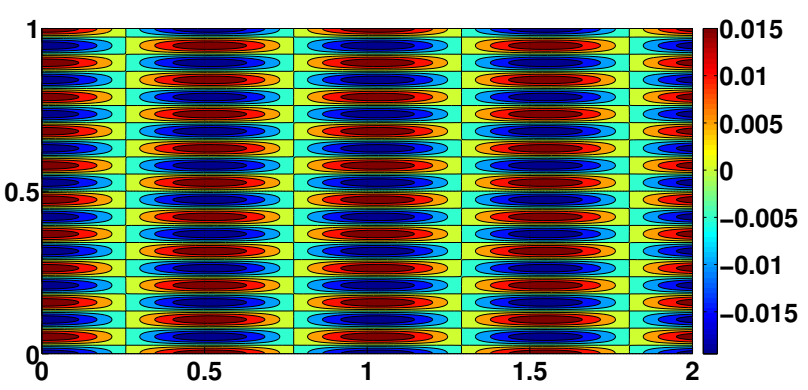

(b)

Figure 25: PUFE solution for the problem of a planewave scattered inside a duct with rigid walls $(k=60, q=12)$. (a) Real component; and (b) Imaginary component.

\section{Concluding remarks}

In this paper, we developed a novel scheme to compute highly oscillatory integrals that appear in the planewave-enriched PUFE scheme to solve the two-dimensional Helmholtz problem. We used the divergence theorem to reduce domain integrals to a set of line integrals and similar domain integrals. Through a recursive procedure these oscillatory integrals were evaluated in terms of just the nodal values of the oscillatory integrands. The new method delivered accuracy to within machine precision for a test example over various simple polygons. Since the derivation was analytical, the approach can be used for arbitrary high values of wave number without any loss in accuracy.

For the Helmholtz problem, the PUFEM offers the advantage of requiring fewer number of degrees of freedom. However, conventionally used interpolatory cubature schemes severely limit the range of wave number that can be treated. The current approach resolves this issue as a whole, since it could deliver accurate results in Sobolev error norms for three benchmark Helmholtz problems. We also showcased the advantages of using rectangular finite elements, which required the recursive scheme to be carried out only once over the reference biunit square element.

A restriction of the proposed scheme is that it is only applicable to FE meshes that consist of elements with straight edges and constant Jacobian in the isoparametric map. In spite of these limitations, the method has appeal since it is suitable for structured Cartesian meshes and unstructured Delaunay meshes that can describe an arbitrary domain, the latter of which is shown in the numerical example of Section 5.3. The present work opens up new avenues for future work. The generalization of the proposed integration scheme to three dimensions and the scope of using techniques based on the divergence theorem to compute other oscillatory integrals of the form (5) are topics that are worth pursuing.

\section{Acknowledgements}

The authors are grateful for the research support through the Program on New Research Initiatives and Interdisciplinary Research at the University of California at Davis. Additional financial support from the National Science Foundation through contract grant CMMI-1334783 to the University of California at Davis is also acknowledged. 


\section{Appendix A. Element stiffness matrix of the biunit square element}

Closed-form expressions are derived of the oscillatory integrals that appear in (26) for the biunit square element $\Omega_{\square}$. We use the components of local wave numbers as presented in Section 4.1.1. The bilinear shape functions in $\Omega_{\square}$ are

$$
N_{i}(\xi, \eta)=\frac{1}{4}\left(1+\xi \xi_{i}\right)\left(1+\eta \eta_{i}\right), \quad(i=1, \ldots, 4) .
$$

These same set of functions are also used as the partition-of-unity functions in the PUFE approximations. Referring to the uniform Cartesian mesh of Fig. 7, the Jacobian matrix corresponding to the isoparametric transformation is

$$
\boldsymbol{J}=\left[\begin{array}{cc}
\frac{\Delta x}{2} & 0 \\
0 & \frac{\Delta y}{2}
\end{array}\right] .
$$

Hence,

$$
\boldsymbol{J}^{-\top} \boldsymbol{J}^{-1}=\boldsymbol{J}^{-2}=\left[\begin{array}{cc}
J_{11}^{-2} & 0 \\
0 & J_{22}^{-2}
\end{array}\right],
$$

where $J_{11}^{-2}=4 /(\Delta x)^{2}$ and $J_{22}^{-2}=4 /(\Delta y)^{2}$.

Now, we obtain expressions for the block matrices in (28). With $N_{i}$ as the FE shape function, $N_{j}$ as the partition-of-unity function, and local planewave enrichment function $W_{n}^{e}$ of the form (4.1.1), we have

$$
\begin{gathered}
\left(\boldsymbol{k}_{\mathrm{FE}-\mathrm{FE}}\right)_{i j}=\int_{\Omega_{\square}}\left\{J_{11}^{-2} \frac{\partial N_{i}}{\partial \xi} \frac{\partial N_{j}}{\partial \xi}+J_{22}^{-2} \frac{\partial N_{i}}{\partial \eta} \frac{\partial N_{j}}{\partial \eta}\right\}|\boldsymbol{J}| d \Omega_{\square}-k^{2} \int_{\Omega_{\square}} N_{i} N_{j}|\boldsymbol{J}| d \Omega_{\square} \\
\quad-i k \int_{\hat{\Gamma}} N_{i} N_{j}|\boldsymbol{J}|_{1 d} d \hat{\Gamma}, \\
\left(\boldsymbol{k}_{\mathrm{PU}-\mathrm{FE}}\right)_{i j}=\int_{\Omega_{\square}}\left\{J_{11}^{-2} \frac{\partial N_{i}}{\partial \xi}\left(\frac{\partial N_{j}}{\partial \xi} W_{n}^{e}+N_{j} \frac{\partial W_{n}^{e}}{\partial \xi}\right)+J_{22}^{-2} \frac{\partial N_{i}}{\partial \eta}\left(\frac{\partial N_{j}}{\partial \eta} W_{n}^{e}+N_{j} \frac{\partial W_{n}^{e}}{\partial \eta}\right)\right\}|\boldsymbol{J}| d \Omega_{\square} \\
-k^{2} \int_{\Omega_{\square}} N_{i} N_{j} W_{n}^{e}|\boldsymbol{J}| d \Omega_{\square}-i k \int_{\hat{\Gamma}} N_{i} N_{j} W_{n}^{e}|\boldsymbol{J}|_{1 d} d \hat{\Gamma}, \\
\left(\boldsymbol{k}_{\mathrm{FE}-\mathrm{PU}}\right)_{i j}=\left(\boldsymbol{k}_{\mathrm{PU}-\mathrm{FE}}^{*}\right)_{j i}=\int_{\Omega_{\square}}\left\{J_{11}^{-2} \frac{\partial N_{j}}{\partial \xi}\left(\frac{\partial N_{i}}{\partial \xi} W_{n}^{* e}+N_{i} \frac{\partial W_{n}^{* e}}{\partial \xi}\right)\right. \\
\left.+J_{22}^{-2} \frac{\partial N_{j}}{\partial \eta}\left(\frac{\partial N_{i}}{\partial \eta} W_{n}^{* e}+N_{i} \frac{\partial W_{n}^{* e}}{\partial \eta}\right)\right\}|\boldsymbol{J}| d \Omega_{\square} \\
-k^{2} \int_{\Omega_{\square}} N_{j} N_{i} W_{n}^{* e}|\boldsymbol{J}| d \Omega_{\square}-i k \int N_{j} N_{i} W_{n}^{* e}|\boldsymbol{J}|_{1 d} d \hat{\Gamma},
\end{gathered}
$$




$$
\begin{aligned}
\left(\boldsymbol{k}_{\mathrm{PU}-\mathrm{PU}}\right)_{i j}= & \int_{\Omega_{\square}}\left\{J_{11}^{-2}\left(W_{r}^{* e} \frac{\partial N_{i}}{\partial \xi}+\frac{\partial W_{r}^{* e}}{\partial \xi} N_{i}\right)\left(\frac{\partial N_{j}}{\partial \xi} W_{s}^{e}+N_{j} \frac{\partial W_{s}^{e}}{\partial \xi}\right)\right. \\
& \left.+J_{22}^{-2}\left(W_{r}^{* e} \frac{\partial N_{i}}{\partial \eta}+\frac{\partial W_{r}^{* e}}{\partial \eta} N_{i}\right)\left(\frac{\partial N_{j}}{\partial \eta} W_{s}^{e}+N_{j} \frac{\partial W_{s}^{e}}{\partial \eta}\right)\right\}|\boldsymbol{J}| d \Omega_{\square} \\
& -k^{2} \int_{\Omega_{p}} N_{i} N_{j} W_{r}^{* e} W_{s}^{e}|\boldsymbol{J}| d \Omega_{\square}-i k \int_{\hat{\Gamma}} N_{i} N_{j} W_{r}^{* e} W_{s}^{e}|\boldsymbol{J}|_{1 d} d \hat{\Gamma}
\end{aligned}
$$

for $n, r$, and $s=0, \ldots, q-1$.

\section{Appendix B. Element stiffness matrix for the reference triangle}

Consider the unit triangular element $\Omega_{\Delta}$ shown in Fig. 8 . The linear shape functions are

$$
N_{1}=1-\xi-\eta, \quad N_{2}=\xi, \quad N_{3}=\eta .
$$

Unlike the case of a rectangular element, the Jacobian matrix corresponding to the isoparametric transformation in Fig. 8 is non-diagonal, and is given by

$$
\boldsymbol{J}=\left[\begin{array}{cc}
x_{2}^{e}-x_{1}^{e} & y_{2}^{e}-y_{1}^{e} \\
x_{3}^{e}-x_{1}^{e} & y_{3}^{e}-y_{1}^{e}
\end{array}\right] \equiv\left[\begin{array}{cc}
J_{11} & J_{12} \\
J_{21} & J_{22}
\end{array}\right]
$$

Hence,

$$
\boldsymbol{J}^{-\top} \boldsymbol{J}^{-1}=\frac{1}{|\boldsymbol{J}|^{2}}\left[\begin{array}{cc}
\left(J_{21}^{2}+J_{22}^{2}\right) & -\left(J_{11} J_{21}+J_{12} J_{22}\right) \\
-\left(J_{11} J_{21}+J_{12} J_{22}\right) & \left(J_{11}^{2}+J_{12}^{2}\right)
\end{array}\right]=\left[\begin{array}{cc}
J_{11}^{-2} & J_{12}^{-2} \\
J_{21}^{-2} & J_{22}^{-2}
\end{array}\right]
$$

is also non-diagonal.

For a triangular element $\Omega_{e}$ mapped to $\Omega_{\Delta}$, expressions for the block matrices in (28) are obtained. Proceeding along similar lines as in Appendix A, we have

$$
\begin{aligned}
&\left(\boldsymbol{k}_{\mathrm{FE}-\mathrm{FE}}\right)_{i j}=\int_{\Omega_{\triangle}}\left\{J_{11}^{-2} \frac{\partial N_{i}}{\partial \xi} \frac{\partial N_{j}}{\partial \xi}+J_{12}^{-2} \frac{\partial N_{i}}{\partial \xi} \frac{\partial N_{j}}{\partial \eta}+J_{21}^{-2} \frac{\partial N_{i}}{\partial \eta} \frac{\partial N_{j}}{\partial \xi}+J_{22}^{-2} \frac{\partial N_{i}}{\partial \eta} \frac{\partial N_{j}}{\partial \eta}\right\}|\boldsymbol{J}| d \Omega_{\Delta} \\
&-k_{\Omega_{\Delta}}^{2} N_{i} N_{j}|\boldsymbol{J}| d \Omega_{\Delta}-i k \int_{\hat{\Gamma}} N_{i} N_{j}|\boldsymbol{J}|_{1 d} d \hat{\Gamma}, \\
&\left(\boldsymbol{k}_{\mathrm{PU}-\mathrm{FE}}\right)_{i j}= \int_{\Omega_{\Delta}}\left\{J_{11}^{-2} \frac{\partial N_{i}}{\partial \xi}\left(\frac{\partial N_{j}}{\partial \xi} W_{n}+N_{j} \frac{\partial W_{n}}{\partial \xi}\right)+J_{12}^{-2} \frac{\partial N_{i}}{\partial \xi}\left(\frac{\partial N_{j}}{\partial \eta} W_{n}+N_{j} \frac{\partial W_{n}}{\partial \eta}\right)\right. \\
&+J_{21}^{-2} \frac{\partial N_{i}}{\partial \eta}\left(\frac{\partial N_{j}}{\partial \xi} W_{n}+N_{j} \frac{\partial W_{n}}{\partial \xi}\right) \\
&\left.+J_{22}^{-2} \frac{\partial N_{i}}{\partial \eta}\left(\frac{\partial N_{j}}{\partial \eta} W_{n}+N_{j} \frac{\partial W_{n}}{\partial \eta}\right)\right\}|\boldsymbol{J}| d \Omega_{\triangle} \\
&-k^{2} \int_{\Omega_{\Delta}} N_{i} N_{j} W_{n}|\boldsymbol{J}| d \Omega_{\Delta}-i k \int N_{i} N_{j} W_{n}|\boldsymbol{J}|_{1 d} d \hat{\Gamma},
\end{aligned}
$$




$$
\begin{aligned}
\left(\boldsymbol{k}_{\mathrm{FE}-\mathrm{PU}}\right)_{i j}=\left(\boldsymbol{k}_{\mathrm{PU}-\mathrm{FE}}^{*}\right)_{j i}= & \int_{\Omega_{\triangle}}\left\{J_{11}^{-2} \frac{\partial N_{j}}{\partial \xi}\left(\frac{\partial N_{i}}{\partial \xi} W_{n}^{* e}+N_{i} \frac{\partial W_{n}^{* e}}{\partial \xi}\right)+J_{12}^{-2} \frac{\partial N_{j}}{\partial \xi}\left(\frac{\partial N_{i}}{\partial \eta} W_{n}^{* e}+N_{i} \frac{\partial W_{n}^{* e}}{\partial \eta}\right)\right. \\
& +J_{21}^{-2} \frac{\partial N_{j}}{\partial \eta}\left(\frac{\partial N_{i}}{\partial \xi} W_{n}^{* e}+N_{i} \frac{\partial W_{n}^{* e}}{\partial \xi}\right) \\
& \left.+J_{22}^{-2} \frac{\partial N_{j}}{\partial \eta}\left(\frac{\partial N_{i}}{\partial \eta} W_{n}^{* e}+N_{i} \frac{\partial W_{n}^{* e}}{\partial \eta}\right)\right\}|\boldsymbol{J}| d \Omega_{\Delta} \\
& -k^{2} \int N_{j} N_{i} W_{n}^{* e}|\boldsymbol{J}| d \Omega_{\Delta}-i k \int N_{j} N_{i} W_{n}^{* e}|\boldsymbol{J}|_{1 d} d \hat{\Gamma}, \\
\left(\boldsymbol{k}_{\mathrm{PU}-\mathrm{PU})_{i j}=}\right. & \int_{\Omega_{\Delta}}\left\{J_{11}^{-2}\left(W_{r}^{* e} \frac{\partial N_{i}}{\partial \xi}+\frac{\partial W_{r}^{* e}}{\partial \xi} N_{i}\right)\left(\frac{\partial N_{j}}{\partial \xi} W_{s}^{e}+N_{j} \frac{\partial W_{s}^{e}}{\partial \xi}\right)\right. \\
& +J_{12}^{-2}\left(W_{r}^{* e} \frac{\partial N_{i}}{\partial \xi}+\frac{\partial W_{r}^{* e}}{\partial \xi} N_{i}\right)\left(\frac{\partial N_{j}}{\partial \eta} W_{s}^{e}+N_{j} \frac{\partial W_{s}^{e}}{\partial \eta}\right) \\
& +J_{21}^{-2}\left(W_{r}^{* e} \frac{\partial N_{i}}{\partial \eta}+\frac{\partial W_{r}^{* e}}{\partial \eta} N_{i}\right)\left(\frac{\partial N_{j}}{\partial \xi} W_{s}^{e}+N_{j} \frac{\partial W_{s}^{e}}{\partial \xi}\right) \\
& \left.+J_{22}^{-2}\left(W_{r}^{* e} \frac{\partial N_{i}}{\partial \eta}+\frac{\partial W_{r}^{* e}}{\partial \eta} N_{i}\right)\left(\frac{\partial N_{j}}{\partial \eta} W_{s}^{e}+N_{j} \frac{\partial W_{s}^{e}}{\partial \eta}\right)\right\}|\boldsymbol{J}| d \Omega_{\Delta} \\
& -k^{2} \int_{\Omega_{\Delta}} N_{i} N_{j} W_{r}^{* e} W_{s}^{e}|\boldsymbol{J}| d \Omega_{\Delta}-i k \int N_{i} N_{j} W_{r}^{* e} W_{s}^{e}|\boldsymbol{J}|_{1 d} d \hat{\Gamma} \\
& -\hat{\Gamma}
\end{aligned}
$$

for $n, r$, and $s=0, \ldots, q-1$.

\section{Appendix C. Element stiffness matrix for biunit one-dimensional element}

We consider the biunit linear parent element $\hat{\Gamma}$ shown in Fig. 9 . The linear shape functions are

$$
\left(N_{1 d}\right)_{1}=\frac{1}{2}(1-\xi), \quad\left(N_{1 d}\right)_{2}=\frac{1}{2}(1+\xi) .
$$

We denote by $\boldsymbol{N}_{1 d}=\left[\left(N_{1 d}\right)_{1}\left(N_{1 d}\right)_{2}\right]^{\top}$ and note that the Jacobian $|\boldsymbol{J}|_{1 d}$ of physical element $\Gamma_{e}$ to $\hat{\Gamma}$ transformation is half the element length. As shown in Section 4.1.3, the one-dimensional contribution to the stiffness matrix is

$$
\boldsymbol{k}_{e_{1 d}}=\int_{\hat{\Gamma}}\left[\begin{array}{ll}
\boldsymbol{N}_{1 d} \boldsymbol{N}_{1 d}^{\top} & \boldsymbol{N}_{1 d} \boldsymbol{\Phi}_{1 d}^{\top} \\
\boldsymbol{\Phi}_{1 d}^{*} \boldsymbol{N}_{1 d}^{\top} & \boldsymbol{\Phi}_{1 d}^{*} \boldsymbol{\Phi}_{1 d}^{\top}
\end{array}\right]|\boldsymbol{J}|_{1 d} d \hat{\Gamma},
$$

which contain oscillatory integrals over the boundary of the problem domain. Note that these integrals are same as those appearing in the last term of (A.1)-(A.4) and (B.1) $-(B .4)$. We now partition (C.1) as

$$
\boldsymbol{k}_{e_{1 d}}=\left[\begin{array}{c|c}
\left(\boldsymbol{k}_{e_{1 d}}\right)_{\mathrm{FE}-\mathrm{FE}} & \left(\boldsymbol{k}_{e_{1 d}}\right)_{\mathrm{PU}-\mathrm{FE}} \\
\hline\left(\boldsymbol{k}_{e_{1 d}}\right)_{\mathrm{FE}-\mathrm{PU}} & \left(\boldsymbol{k}_{e_{1 d}}\right)_{\mathrm{PU}-\mathrm{PU}}
\end{array}\right] .
$$


Hence, considering a two-node element $\Gamma_{e}$ mapped to $\hat{\Gamma}$, the expressions for the block matrices in (C.2) are obtained. Proceeding along similar lines as in the preceding two sections of the Appendix, we have

$$
\begin{gathered}
{\left[\left(\boldsymbol{k}_{e_{1 d}}\right)_{\mathrm{FE}-\mathrm{FE}}\right]_{i j}=i k \int_{\hat{\Gamma}}\left(N_{1 d}\right)_{i}\left(N_{1 d}\right)_{j}|\boldsymbol{J}|_{1 d} d \hat{\Gamma},} \\
{\left[\left(\boldsymbol{k}_{e_{1 d}}\right)_{\mathrm{PU}-\mathrm{FE}}\right]_{i j}=i k \int_{\hat{\Gamma}}\left(N_{1 d}\right)_{i}\left(N_{1 d}\right)_{j} W_{n}^{e_{1 d}}|\boldsymbol{J}|_{1 d} d \hat{\Gamma},} \\
{\left[\left(\boldsymbol{k}_{e_{1 d}}\right)_{\mathrm{FE}-\mathrm{PU}}\right]_{i j}=\left[\left(\boldsymbol{k}_{e_{1 d}}^{*}\right)_{\mathrm{PU}-\mathrm{FE}}\right]_{j i}=i k \int_{\hat{\Gamma}}\left(N_{1 d}\right)_{i}\left(N_{1 d}\right)_{j} W_{n}^{* e_{1 d}}|\boldsymbol{J}|_{1 d} d \hat{\Gamma},} \\
{\left[\left(\boldsymbol{k}_{e_{1 d}}\right)_{\mathrm{PU}-\mathrm{PU}}\right]_{i j}=i k \int_{\hat{\Gamma}}\left(N_{1 d}\right)_{i}\left(N_{1 d}\right)_{j} W_{r}^{* e_{1 d}} W_{s}^{e_{1 d}}|\boldsymbol{J}|_{1 d} d \hat{\Gamma},}
\end{gathered}
$$

for $n, r$, and $s=0, \ldots,(q-1)$.

\section{Appendix D. Element load vector for biunit one-dimensional element}

Once again, referring to Section 4.1 .3 , the load vector is:

$$
\boldsymbol{f}_{e_{1 d}}=\int_{\hat{\Gamma}} g\left\{\begin{array}{l}
\boldsymbol{N}_{1 d} \\
\boldsymbol{\Phi}_{1 d}^{*}
\end{array}\right\}|\boldsymbol{J}|_{1 d} d \hat{\Gamma} .
$$

We also partition the load vector in $(\overline{D .1})$ as

$$
f_{e_{1 d}}=\left\{\frac{\left(f_{e_{1 d}}\right)_{\mathrm{FE}}}{\left(f_{e_{1 d}}\right)_{\mathrm{PU}}}\right\},
$$

with components of the form

$$
\begin{gathered}
{\left[\left(\boldsymbol{f}_{e_{1 d}}\right)_{\mathrm{FE}}\right]_{i}=\int_{\hat{\Gamma}}\left(N_{1 d}\right)_{i} g|\boldsymbol{J}|_{1 d} d \hat{\Gamma},} \\
{\left[\left(\boldsymbol{f}_{e_{1 d}}\right)_{\mathrm{PU}}\right]_{i}=\int_{\hat{\Gamma}}\left(N_{1 d}\right)_{i} W_{n}^{* e_{1 d}} g|\boldsymbol{J}|_{1 d} d \hat{\Gamma}}
\end{gathered}
$$

for $n=0, \ldots,(q-1)$.

\section{References}

[1] I. Babuška, F. Ihlenburg, E. T. Paik, S. A. Sauter, A generalized finite element method for solving the Helmholtz equation in two dimensions with minimal pollution, Computer Methods in Applied Mechanics and Engineering 128 (3-4) (1995) 325-359. 
[2] I. Babuška, S. A. Sauter, Is the pollution effect of the fem avoidable for the Helmholtz equation considering high wave numbers?, SIAM Journal on Numerical Analysis 34 (6) (1997) 2392-2423.

[3] F. Ihlenburg, Finite Element Analysis of Acoustic Scattering, Springer-Verlag: New York, 1998.

[4] L. L. Thompson, P. M. Pinsky, A Galerkin least squares finite element method for the twodimensional Helmholtz equation, International Journal for Numerical Methods in Engineering 38 (3) (1995) 371-397.

[5] J. M. Melenk, On generalized finite element methods, Ph.D. thesis, University of Maryland, College Park, MD (1995).

[6] J. M. Melenk, I. Babuška, The partition of unity finite element method: Basic theory and applications, Computer Methods in Applied Mechanics and Engineering 139 (1-4) (1996) 289-314.

[7] I. Babuška, J. M. Melenk, The partition of unity method, International Journal for Numerical Methods in Engineering 40 (4) (1997) 727-758.

[8] T. Strouboulis, I. Babuška, K. Copps, The design and analysis of the generalized finite element method, Computer Methods in Applied Mechanics and Engineering 181 (1-3) (2000) 43-69.

[9] T. Strouboulis, I. Babuška, K. Copps, The generalized finite element method: an example of its implementation and illustration of its performance, International Journal for Numerical Methods in Engineering 47 (8) (2000) 1401-1417.

[10] T. Strouboulis, K. Copps, I. Babuška, The generalized finite element method, Computer Methods in Applied Mechanics and Engineering 190 (32-33) (2001) 408-4193.

[11] O. Cessenat, Application d'une nouvelle formulation variationnelle aux équations d'ondes harmoniques, problémes de Helmholtz 2D et de Maxwell 3D, Ph.D. thesis, Université Paris IX Dauphine (1996).

[12] O. Cessenat, B. Despres, Application of an ultra weak variational formulation of elliptic pdes to the two-dimensional Helmholtz problem, SIAM Journal on Numerical Analysis 35 (1) (1998) 255-299.

[13] C. Farhat, I. Harari, L. P. Franca, The discontinuous enrichment method, Computer Methods in Applied Mechanics and Engineering 190 (48) (2001) 6455-6479.

[14] C. Farhat, I. Harari, U. Hetmaniuk, A discontinuous Galerkin method with Lagrange multipliers for the solution of Helmholtz problems in the mid-frequency regime, Computer Methods in Applied Mechanics and Engineering 192 (11-12) (2003) 1389-1419.

[15] M. Griebel, M. A. Schweitzer, A particle-partition of unity method for the solution of elliptic, parabolic, and hyperbolic PDEs, SIAM Journal on Scientific Computing 22 (3) (2000) 853890. 
[16] M. A. Schweitzer, A Parallel Multilevel Partition of Unity Method for Elliptic Partial Differential Equations, Vol. 29 of Lecture Notes in Computational Science and Engineering, Springer-Verlag: New York, 2003.

[17] R. Hiptmair, A. Moiola, I. Perugia, Plane wave discontinuous Galerkin methods for the $2 \mathrm{~d}$ Helmholtz equation: analysis of the p-version, SIAM Journal on Numerical Analysis 49 (1) (2011) 264-284.

[18] I. Perugia, P. Pietra, A. Russo, A plane wave virtual element method for the Helmholtz problem, ESAIM: Mathematical Modelling and Numerical Analysis 50 (3) (2016) 783-808, Available as arXiv preprint: http://arxiv.org/abs/1505.04965.

[19] T. Strouboulis, I. Babuška, R. . Hidajat, The generalized finite element method for Helmholtz equation: theory, computation, and open problems, Computer Methods in Applied Mechanics and Engineering 195 (37-40) (2006) 4711-4731.

[20] O. Laghrouche, P. Bettess, Short wave modelling using special finite elements, Journal of Computational Acoustics 8 (1) (2000) 189-210.

[21] L. N. G. Filon, On a quadrature formula for trigonometric integrals, Proceedings of the Royal Society of Edinburgh 49 (1928) 38-47.

[22] E. A. Flinn, A modification of Filon's method of numerical integration, Journal of the ACM 7 (4) (1960) 181-184.

[23] D. Levin, Procedures for computing one- and two-dimensional integrals of functions with rapid irregular oscillations, Mathematics of Computation 38 (158) (1982) 531-538.

[24] G. A. Evans, J. R. Webster, A comparison of some methods for the evaluation of highly oscillatory integrals, Journal of Computational and Applied Mathematics 112 (1-2) (1999) $55-69$.

[25] A. Iserles, I. S. P. Nørest, Efficient quadrature of highly oscillatory integrals using derivatives, Proceedings of the Royal Society of London. Series A, Mathematical and Physical Sciences 461 (2057) (2005) 1383-1399.

[26] S. Olver, Numerical approximation of highly oscillatory integrals, Ph.D. thesis, University of Cambridge, Cambridge, UK (2008).

[27] O. Laghrouche, P. Bettess, R. J. Astley, Modelling of short wave diffraction problems using approximating systems of plane waves, International Journal for Numerical Methods in Engineering 54 (10) (2002) 1501-1533.

[28] P. Ortiz, E. Sanchez, An improved partition of unity finite element model for diffraction problems, International Journal for Numerical Methods in Engineering 50 (12) (2001) 2727 2740 .

[29] P. Bettess, J. Shirron, O. Laghrouche, B. Peseux, R. Sugimoto, J. Trevelyan, A numerical integration scheme for special finite elements for the Helmholtz equation, International Journal for Numerical Methods in Engineering 56 (4) (2003) 531-552. 
[30] J. B. Lasserre, Integration on a convex polytope, Proceedings of the American Mathematical Society 126 (8) (1998) 2433-2441.

[31] E. B. Chin, J. B. Lasserre, N. Sukumar, Numerical integration of homogeneous functions on convex and nonconvex polygons and polyhedra, Computational Mechanics 56 (6) (2015) 967-981.

[32] R. J. Astley, P. Gamallo, Special short wave elements for flow acoustics, Computer Methods in Applied Mechanics and Engineering 194 (2-5) (2005) 341-353.

[33] P. Gamallo, R. J. Astley, The partition of unity finite element method for short wave acoustic propagation on non-uniform potential flows, International Journal for Numerical Methods in Engineering 65 (3) (2006) 425-444.

[34] T. Huttunen, P. Gamallo, R. J. Astley, Comparison of two wave element methods for the Helmholtz problem, Communications in Numerical Methods in Engineering 25 (1) (2009) $35-52$. 